\title{
OPTIMAL COORDINATED MOTIONS OF MULTIPLE AGENTS MOVING ON A PLANE *
}

\author{
JIANGHAI HU ${ }^{\dagger}$, MARIA PRANDINI ${ }^{\ddagger}$, AND SHANKAR SASTRY§
}

\begin{abstract}
We address the problem of optimal coordinated motions of multiple agents moving in the same planar region. The agents' motions must satisfy a separation constraint throughout the encounter to be conflict-free. The objective is to determine the conflict-free maneuvers (motions) with the least combined energy, while taking into account the fact that agents may have different priorities. A formal classification of conflict-free maneuvers into homotopy types is introduced by using their braid representation. Various local and global optimality conditions are derived through variational analysis in the presence of the separation constraint. In the case of two agents, these optimality conditions allow us to construct the optimal maneuvers geometrically. For the general multi-agent case, a convex optimization algorithm is proposed to compute within each homotopy type a solution to the optimization problem restricted to the class of multi-legged maneuvers. Since the number of types grows explosively with the number of agents, a stochastic algorithm is suggested as the "type chooser", thus leading to a randomized optimization algorithm.
\end{abstract}

Key words. cooperative motion planning, braids, calculus of variation with constraints, convex optimization.

AMS subject classifications. 65K10, 93C $85,90 \mathrm{C} 25$.

1. Introduction. In this paper, the problem of designing coordinated maneuvers for multiple agents moving on a plane is studied. The joint maneuver has to be chosen so as to guide each agent from its starting position to its target position, while avoiding conflicts, that is, situations where the Euclidean distance between any two agents is smaller than some fixed threshold $R>0$. Among all the conflict-free joint maneuvers, we aim at determining the one with the least overall cost. Here the cost of a single agent's maneuver is its energy, and the overall cost is a weighted sum of the maneuver energies of all individual agents, with the weights representing the priorities of the agents. A precise formulation of the problem is given in Section 3 .

This problem is of great interest since it is actually encountered in many different practical areas. For example, in the air traffic control (ATC) context, aircraft flying at the same altitude must maintain a minimal horizontal separation $R$ of at least 3 nautical miles (nmi) inside the terminal radar approach control facilities and $5 \mathrm{nmi}$ in the en-route airspace ([35]). In this case, the energy is closely related to practical aspects such as travel distance, fuel consumption, passenger comfort, etc. Numerous approaches have been proposed in the literature on aircraft conflict resolution, including optimal control theory ([5]), semidefinite programming ([10]), sequential quadratic programming $([29])$, game theory $([39,40])$, parallel coordinates representation $([19])$, genetic algorithms ([28]), to name a few. Readers are referred to [15, 23] for a survey on aircraft conflict resolution. Similar problems have been studied in

${ }^{*}$ Part of the results in this paper have appeared in $[12,14,17]$. These references are available in electronic format upon request. This research has been supported by DARPA under Grant No. F33615-98-C-3614, by the National Science Foundation under Grant No. EIA-0122599, and by MIUR under the project "New techniques for the identification and adaptive control of industrial systems."

${ }^{\dagger}$ Department of Electrical Engineering and Computer Sciences, University of California at Berkeley, Berkeley, CA 94720 (jianghai@robotics.eecs.berkeley.edu).

‡Dipartimento di Elettronica e Informazione, Politecnico di Milano, Piazza Leonardo da Vinci 32, 20133 Milano, Italy (prandini@elet.polimi.it).

$\S$ Department of Electrical Engineering and Computer Sciences, University of California at Berkeley, Berkeley, CA 94720 (sastry@robotics.eecs.berkeley.edu). 
other transportation systems as well, such as [30].

The problem of optimal multi-agent coordinated motions also finds applications in robotics. For example, for multiple cooperating mobile robots moving in a common workspace, the requirement that there is no collision among them can be reformulated as that their joint maneuver is conflict-free, with $R$ being twice the robot radius. The literature on the general problem of robot motion planning with static or dynamic obstacles is vast (see e.g. [4, 9, 11, 24, 38], and the survey [18]), and it is impossible to survey them in this paper. Here we limit our review to those contributions more relevant to our work. A large portion of the treatments focus on the feasibility and the algorithmic complexity aspects of the problem. Some of them indeed deal with multiple robots case using certain optimality criteria. To name a few, [5] studies the problem of time-optimal cooperative motions of multiple Dubin vehicles moving at constant speed with bounded curvature, while in [25], each robot minimizes its own independent cost function by using techniques from multi-objective optimization and game theory. [7] addresses the problem of optimal motion planning for multiple nonholonomic manipulators transporting a grasped object.

The distinguishing feature of our approach to coordinated motion planning consists in the interpretation of maneuvers as braids. Besides giving a complete homotopic classification of conflict-free maneuvers, this also provides us insights on the derivation of optimality conditions. Although the space-time representation of motions is not new in the literature (see e.g. $[9,37]$ ), to our knowledge, however, it has never been used to such an extent in the optimality analysis of coordinated motions.

Due to the many different interpretations of conflict-free maneuvers (not only as braids, but also as, e.g., solutions to mechanical systems or geodesics in a manifold with boundary), many of the results in this paper can be derived in more than one way. For example, some of the local optimality conditions in Section 3 can be derived by using the symmetry reduction method in $[3,26]$. In most cases, we choose our approaches with an emphasis on their geometric appealing and their relevancy to the braid point of view. As a result, they may not always be the most elegant and efficient ones. In addition, although we focus exclusively on the case when the state space is $\mathbb{R}^{2}$, extensions to general state spaces are possible $([16])$. These possible extensions, as well as the remaining open issues, will be pointed out in the paper wherever possible.

This paper is organized as follows. In Section 2, we introduce a formal classification of conflict-free maneuvers into homotopy types by using the notion of pure braids group. Inspired by the braid representation of conflict-free maneuvers, we define various transformations of joint maneuvers that preserve the minimum separation condition. Such transformations are used in the variational analysis in Section 3 to derive local and global necessary conditions on optimal conflict-free maneuvers. In particular, the optimal conflict-free maneuvers for the two-agent case are derived in Section 3.3. Two mechanical interpretations of the problem are given in Section 3.8.

As the number of agents increases, it is difficult in practice to derive analytically the optimal conflict-free maneuvers. By focusing on those maneuvers specified by a set of waypoints, we are able to use convex optimization techniques to obtain multilegged approximated solutions to the constrained optimization problem within each homotopy type (Section 4). A stochastic algorithm is proposed in Section 4.4 to address the problem of selecting the homotopy type, thus leading to a randomized convex optimization algorithm.

The paper is concluded in Section 5 with some general remarks and the outline of some possible extensions of this research. 
2. Classification of conflict-free maneuvers. In this section, we introduce a qualitative classification of conflict-free maneuvers involving multiple agents. Roughly speaking, two conflict-free maneuvers are classified as of the same "type" if there exists a continuous conflict-free deformation of one to the other. Hence switching between different types cannot be done smoothly without causing a conflict.

Consider $n$ agents (numbered from 1 to $n$ ) moving in $\mathbb{R}^{2}$, where each agent, say agent $i$, starts at position $a_{i} \in \mathbb{R}^{2}$ at time $t_{0}$, and ends in position $b_{i} \in \mathbb{R}^{2}$ at time $t_{f}$. Let $T \triangleq\left[t_{0}, t_{f}\right]$ be the time interval of the encounter. Denote by $\mathbf{P}_{i} \triangleq\left\{\alpha_{i} \in\right.$ $\left.C\left(T, \mathbb{R}^{2}\right): \alpha_{i}\left(t_{0}\right)=a_{i}, \alpha_{i}\left(t_{f}\right)=b_{i}\right\}$ the set of maneuvers for agent $i$ consisting of all the continuous maps from $T$ to $\mathbb{R}^{2}$ that take the values $a_{i}$ and $b_{i}$ at times $t_{0}$ and $t_{f}$, respectively. Set $\mathbf{P}(\mathbf{a}, \mathbf{b}) \triangleq \prod_{i=1}^{n} \mathbf{P}_{i}$, where $\mathbf{a}=\left(a_{1}, \cdots, a_{n}\right)$ and $\mathbf{b}=\left(b_{1}, \cdots, b_{n}\right)$. Each element $\alpha=\left(\alpha_{1}, \cdots, \alpha_{n}\right) \in \mathbf{P}(\mathbf{a}, \mathbf{b})$ is called a joint maneuver ( $n$-maneuver or simply maneuver when there is no ambiguity) for the $n$-agent system. The minimum separation over encounter (MSE) for a joint maneuver $\alpha$ is defined to be the minimum Euclidean distance between any pair of agents during the whole time interval $T$, i.e.,

$$
\Delta(\alpha) \triangleq \min _{1 \leq i<j \leq n} \inf _{t \in T}\left\|\alpha_{i}(t)-\alpha_{j}(t)\right\|
$$

The set of conflict-free maneuvers is then defined as

$$
\mathbf{P}(R, \mathbf{a}, \mathbf{b}) \triangleq\{\alpha \in \mathbf{P}(\mathbf{a}, \mathbf{b}): \Delta(\alpha)>R\},
$$

where $R$ is a positive number representing, for example, the radius of the protection zone surrounding an aircraft or twice the radius of a circular robot. We assume that the minimum distance between any pair of starting positions in the $n$-tuple $\left\langle a_{i}\right\rangle_{i=1}^{n}$ and any pair of ending positions in the $n$-tuple $\left\langle b_{i}\right\rangle_{i=1}^{n}$ is strictly greater than $R$, so that $\mathbf{P}(R, \mathbf{a}, \mathbf{b})$ is nonempty.

We distinguish different maneuvers in $\mathbf{P}(R, \mathbf{a}, \mathbf{b})$ according to the following equivalence relation.

Definition 2.1 ( $R$-homotopy). Two conflict-free maneuvers in $\mathbf{P}(R, \mathbf{a}, \mathbf{b})$ are $R$-homotopic if there exists a continuous deformation of one to the other in $\mathbf{P}(R, \mathbf{a}, \mathbf{b})$, or equivalently, if there exists a continuous deformation of one to the other in $\mathbf{P}(\mathbf{a}, \mathbf{b})$ such that the joint maneuvers obtained throughout the deformation are conflict-free.

The objective of this section is to characterize the structure of the equivalence classes of $\mathbf{P}(R, \mathbf{a}, \mathbf{b})$ induced by the $R$-homotopy relation. With this purpose in mind, we now recall the concept of braids $([6,32])$.

Definition 2.2 (Braids). A braid joining $\mathbf{a}=\left(a_{1}, \cdots, a_{n}\right)$ to $\mathbf{b}=\left(b_{1}, \cdots, b_{n}\right)$ is an $n$-tuple $\left\langle\gamma_{i}\right\rangle_{i=1}^{n}$ of continuous curves in $\mathbb{R}^{2} \times T \subset \mathbb{R}^{3}$ satisfying the following conditions:

- Each point $\left(a_{i}, t_{0}\right), i=1, \cdots, n$, is joined by exactly one curve in $\left\langle\gamma_{i}\right\rangle_{i=1}^{n}$ to one of the points $\left(b_{j}, t_{f}\right), 1 \leq j \leq n$;

- The plane $t=\tau$ intersects each curve at exactly one point for all $\tau \in T$;

- $\gamma_{i} \cap \gamma_{j}=\emptyset$ whenever $i \neq j$.

In the following, we shall occasionally use the term $n$-braid to indicate the number of curves in the braid. The set of all braids joining $\mathbf{a}$ to $\mathbf{b}$ is denoted by $\mathbf{B}(\mathbf{a}, \mathbf{b})$. If $i$ and $j$ are required to be identical in the first condition of Definition 2.2, the corresponding braid is called a pure braid. The set of all pure braids joining $\mathbf{a}$ to $\mathbf{b}$ is denoted by $\mathbf{P B}(\mathbf{a}, \mathbf{b})$. An example of a pure 3-braid is shown in the right-hand-side of Fig. 2.1.

There is a simple equivalence relation defined on $\mathbf{B}(\mathbf{a}, \mathbf{b})$ and hence on $\mathbf{P B}(\mathbf{a}, \mathbf{b})$ as well $([32])$. 

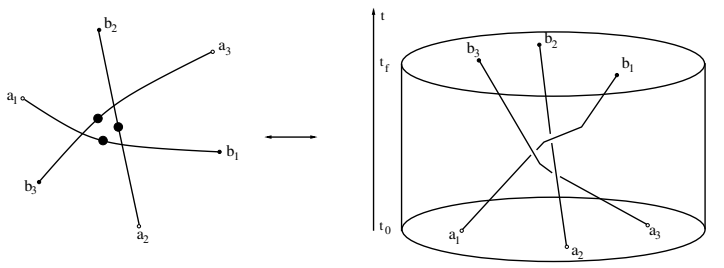

FIG. 2.1. A 3-maneuver in $\mathbf{P}(0, \mathbf{a}, \mathbf{b})$ and its braid representation.

Definition 2.3 (String isotopy). Two braids in $\mathbf{B}(\mathbf{a}, \mathbf{b})$ are said to be string isotopic if the $n$ curves of one of them can be continuously deformed to those of the other such that the $n$ curves in $\mathbb{R}^{2} \times T$ obtained throughout the deformation satisfy all the conditions in Definition 2.2.

The reason for introducing the notion of braids is that there exists a very natural one-to-one correspondence between joint maneuvers in $\mathbf{P}(0, \mathbf{a}, \mathbf{b})$ and pure braids in $\mathbf{P B}(\mathbf{a}, \mathbf{b})$. To see this, for each joint maneuver $\alpha=\left(\alpha_{1}, \cdots, \alpha_{n}\right) \in \mathbf{P}(0, \mathbf{a}, \mathbf{b})$, let $\hat{\alpha}_{i}$ be the curve in $\mathbb{R}^{2} \times T$ joining $\left(a_{i}, t_{0}\right)$ to $\left(b_{i}, t_{f}\right)$ defined as the image of the map $t \mapsto\left(\alpha_{i}(t), t\right), t \in T$. Then, it is clear from the definition of $\mathbf{P}(0, \mathbf{a}, \mathbf{b})$ that the $n$-tuple $\left\langle\hat{\alpha}_{i}\right\rangle_{i=1}^{n}$ of curves is indeed a pure braid in $\mathbf{P B}(\mathbf{a}, \mathbf{b})$, which we shall denote by $\hat{\alpha}$ (see Fig. 2.1 for a 3-maneuver in $\mathbf{P}(0, \mathbf{a}, \mathbf{b})$ and its braid representation). The map $\alpha \mapsto \hat{\alpha}$ can be verified to be a bijection between $\mathbf{P}(0, \mathbf{a}, \mathbf{b})$ and $\mathbf{P B}(\mathbf{a}, \mathbf{b})$. Furthermore, the following result is an immediate consequence of the above definitions.

Proposition 2.4 (Equivalence of 0-homotopy and string isotopy). $\alpha$ and $\beta \in$ $\mathbf{P}(0, \mathbf{a}, \mathbf{b})$ are 0 -homotopic if and only if $\hat{\alpha}$ and $\hat{\beta}$ are string isotopic in $\mathbf{P B}(\mathbf{a}, \mathbf{b})$.

As a result of Proposition 2.4, there is a one-to-one correspondence between the 0-homotopy classes of $\mathbf{P}(0, \mathbf{a}, \mathbf{b})$ and the (string) isotopy classes of $\mathbf{P B}(\mathbf{a}, \mathbf{b})$.

We next show that the isotopy classes of braids with identical starting and ending points, say $\mathbf{B}(\mathbf{a}, \mathbf{a})$, form a group under a suitable product operation. For each $\hat{\alpha} \in$ $\mathbf{B}(\mathbf{a}, \mathbf{b})$ and $\hat{\beta} \in \mathbf{B}(\mathbf{b}, \mathbf{c})$, define the product $\hat{\gamma} \triangleq \hat{\alpha} \cdot \hat{\beta}$ as the braid $\hat{\gamma} \in \mathbf{B}(\mathbf{a}, \mathbf{c})$ obtained by first concatenating the $n$ curves of $\hat{\alpha}$ with those of $\hat{\beta}$, and then renormalizing the $t$ axis linearly such that the resultant $n$ curves connect $\left\langle\left(a_{i}, t_{0}\right)\right\rangle_{i=1}^{n}$ to $\left\langle\left(c_{i}, t_{f}\right)\right\rangle_{i=1}^{n}$ via $\left\langle\left(b_{i}, \frac{t_{0}+t_{f}}{2}\right)\right\rangle_{i=1}^{n}$. Note that the ending points of $\hat{\alpha}$ and the starting points of $\hat{\beta}$ have to coincide for the product to be well defined. It can be easily checked that this product operation preserves string isotopy, i.e., if $\hat{\alpha}^{\prime}$ is string isotopic to $\hat{\alpha}$ in $\mathbf{B}(\mathbf{a}, \mathbf{b})$ and $\hat{\beta}^{\prime}$ is string isotopic to $\hat{\beta}$ in $\mathbf{B}(\mathbf{b}, \mathbf{c})$, then $\hat{\alpha}^{\prime} \cdot \hat{\beta}^{\prime}$ is string isotopic to $\hat{\alpha} \cdot \hat{\beta}$ in $\mathbf{B}(\mathbf{a}, \mathbf{c})$. Therefore, it induces a product operation on the isotopy classes of braids. This induced product operation makes the isotopy classes of $\mathbf{B}(\mathbf{a}, \mathbf{a})$ into a group, with the inverse operation being the reflection of the $n$ curves across the plane $t=\frac{t_{0}+t_{f}}{2}$. We denote this group by $\mathbf{B}_{n}$. Similarly the isotopy classes of pure braids $\mathbf{P B}(\mathbf{a}, \mathbf{a})$ form under the same induced product operation a group, which we denote by $\mathbf{P} \mathbf{B}_{n} . \mathbf{P B}_{n}$ is a normal subgroup of $\mathbf{B}_{n}$. Readers are referred to [12] or [32] for a detailed derivation of the above claims.

Now if we fix a braid $\hat{\beta}$ in $\mathbf{P B}(\mathbf{b}, \mathbf{a})$, then $\hat{\alpha} \mapsto \hat{\alpha} \cdot \hat{\beta}$ defines a map from $\mathbf{P B}(\mathbf{a}, \mathbf{b})$ to $\mathbf{P B}(\mathbf{a}, \mathbf{a})$. Since this map preserves string isotopy, it induces a map from the isotopy classes of $\mathbf{P B}(\mathbf{a}, \mathbf{b})$ to the isotopy classes of $\mathbf{P B}(\mathbf{a}, \mathbf{a})$, i.e., $\mathbf{P B}_{n}$. The induced map is easily verified to be a bijection. This fact combined with the result in Proposition 2.4 implies that there exists a bijection between the 0-homotopy classes of $\mathbf{P}(0, \mathbf{a}, \mathbf{b})$ and the elements of $\mathbf{P B}$. 

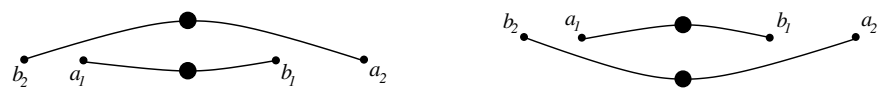

FIG. 2.2. 2-agent encounter. Left: Maneuver 1 - Right: Maneuver 2.

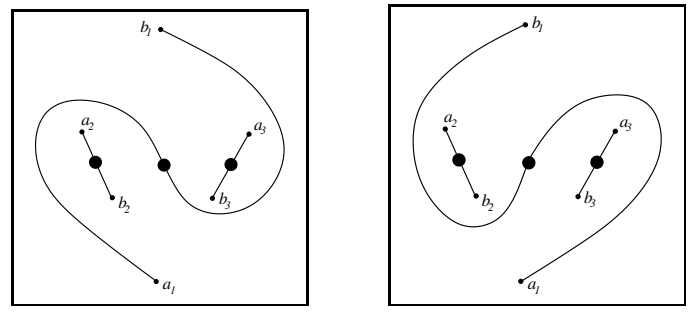

FIG. 2.3. Two 3-maneuvers with the same turning angle but belonging to different types.

The above conclusions remain valid for the case of an arbitrary $R>0$. Hence,

TheOREM 2.5 (Classification of conflict-free $n$-maneuvers). The $R$-homotopy classes of conflict-free maneuvers in $\mathbf{P}(R, \mathbf{a}, \mathbf{b})$ have a one-to-one correspondence with the elements of the group of pure $n$-braids $\mathbf{P} \mathbf{B}_{n}$.

In Remark 1 of Section 3, we give an alternative interpretation of the above result. For a discussion on the use of braid groups to classify motions on a graph, see [1].

The group $\mathbf{P} \mathbf{B}_{n}$ is described by a set of generators together with a set of relations defined on them $([32,33])$. Therefore, Theorem 2.5 completely characterizes the structure of the homotopy types of conflict-free maneuvers for $n$-agent encounters. On the other hand, the characterization is unsatisfactory in practical terms since the description of $\mathbf{P} \mathbf{B}_{n}$ is very complicated. However, when $n$ is small, the result in Theorem 2.5 may have simple interpretations. Consider for example the 2-agent encounter shown in Fig. 2.2. Theorem 2.5 says that each maneuver in $\mathbf{P}(0, \mathbf{a}, \mathbf{b})$ is 0-homotopic to maneuver 1 , or maneuver 2 , or one of the following two maneuvers:

- Maneuver 1 followed by the motions where agent 2 stays at $b_{2}$, and agent 1 starts from $b_{1}$, circles around agent 2 counterclockwise $k$ times for some integer $k \geq 1$ and returns to $b_{1}$.

- Maneuver 2 followed by the motions where agent 2 stays at $b_{2}$, agent 1 starts from $b_{1}$, circles around agent 2 clockwise $k$ times for some integer $k \geq 1$ and returns to $b_{1}$.

The angle one agent turns with respect to the other during $T$ plays a decisive role in determining the homotopy type of the conflict-free 2-maneuvers. Maneuver 1 and maneuver 2 are representatives of the only two types for which the absolute values of this angle do not exceed $360^{\circ}$. We shall call such types fundamental. Then there are exactly two fundamental types for any 2-agent encounter.

It is tempting to extend this definition to the $n$-agent case, and conclude that there are exactly $2^{\frac{n(n-1)}{2}}$ fundamental types of conflict-free maneuvers, since there are two fundamental types for each of the $\frac{n(n-1)}{2}$ agent pairs. Unfortunately this is not the case. Shown in Fig. 2.3 are the plots of two conflict-free maneuvers for a 3 -agent encounter that have the same turning angle within the range $\left(-360^{\circ}, 360^{\circ}\right)$ between any pair of agents, but in fact belong to different types. 
3. Optimal conflict-free maneuvers. In this section, the problem of finding "optimal" conflict-free maneuvers for multi-agent encounters is formulated and studied. To ensure that the problem is well defined and admits a solution, we modify some of the notations introduced in the previous section. In particular, the set of maneuvers for agent $i, \mathbf{P}_{i}$, is redefined to be the set of all continuous and piecewise $C^{2}$ maps $^{1}$ from $T$ to $\mathbb{R}^{2}$ that take the values $a_{i}$ and $b_{i}$ at times $t_{0}$ and $t_{f}$, respectively. The set of joint maneuvers $\mathbf{P}(\mathbf{a}, \mathbf{b})$ and the MSE $\Delta(\alpha), \alpha \in \mathbf{P}(\mathbf{a}, \mathbf{b})$, are defined as in Section 2 , whereas $\mathbf{P}(R, \mathbf{a}, \mathbf{b})$ is redefined to be the set of all joint maneuvers with a MSE greater than or equal to $R$. Note that the results in Section 2 on the qualitative classification of conflict-free maneuvers still hold for the newly defined $\mathbf{P}(R, \mathbf{a}, \mathbf{b})$ by the compactness of $T$.

3.1. Statement of the problem. Consider a maneuver of a single agent, say $\alpha_{i} \in \mathbf{P}_{i}, i \in\{1, \cdots, n\}$. The energy of $\alpha_{i}$ is defined as

$$
J\left(\alpha_{i}\right)=\frac{1}{2} \int_{t_{0}}^{t_{f}}\left\|\dot{\alpha}_{i}(t)\right\|^{2} d t
$$

Let $L\left(\alpha_{i}\right)$ be the arc length of the curve $\alpha_{i}$, i.e., $L\left(\alpha_{i}\right)=\int_{t_{0}}^{t_{f}}\left\|\dot{\alpha}_{i}(t)\right\| d t$. Then the application of Cauchy-Schwartz inequality to equation (3.1) yields ([31]):

$$
J\left(\alpha_{i}\right) \geq \frac{1}{2} \frac{L\left(\alpha_{i}\right)^{2}}{\left(t_{f}-t_{0}\right)}
$$

where the equality holds if and only if $\left\|\dot{\alpha}_{i}(t)\right\|$ is constant. This implies that if agent $i$ is forced to move along some fixed curve and if we ignore the presence of other agents temporarily, then of all the different parameterizations, the one with a constant speed has the minimal energy, and the minimal energy is proportional to the square of the curve length. Therefore, in the presence of static obstacles, the maneuver of agent $i$ with the least energy between two points is the shortest curve between them parameterized proportionally to the arc length. In particular, if there are no obstacles, the energy-minimizing maneuver of agent $i$ is the constant speed motion along the line segment from $a_{i}$ to $b_{i}$. It follows from this discussion that the energy-minimizing maneuvers tend to be straighter and smoother, which has practical implications, for example, in terms of passenger comfort, brake erosion, fuel consumption, etc.

The $\mu$-energy of a joint maneuver $\alpha=\left(\alpha_{1}, \ldots, \alpha_{n}\right) \in \mathbf{P}(\mathbf{a}, \mathbf{b})$ is defined as

$$
J_{\mu}(\alpha) \triangleq \sum_{i=1}^{n} \mu_{i} J\left(\alpha_{i}\right)
$$

where $\mu_{1}, \ldots, \mu_{n}$ are $n$ positive numbers adding up to 1 (i.e. $\sum_{i=1}^{n} \mu_{i}=1$ ) representing the priorities of the agents.

Our goal is to find the conflict-free maneuver with the least $\mu$-energy, i.e.,

$$
\text { minimize } J_{\mu}(\alpha) \text { subject to } \alpha \in \mathbf{P}(R, \mathbf{a}, \mathbf{b}) \text {. }
$$

If $\alpha$ is required to belong to a certain type in $\mathbf{P}(R, \mathbf{a}, \mathbf{b})$, then we get a restricted version of problem (3.4). All the necessary conditions obtained in this section remain valid for the restricted problem, with the only exception of Proposition 3.8.

\footnotetext{
${ }^{1}$ Piecewise $C^{2}$ means that there is a finite subdivision of $T$ such that the map is continuously differentiable till the second order on each (open) subinterval. In the sequel when we use $\dot{\alpha}_{i}(t), \ddot{\alpha}_{i}(t)$, we shall mean at those $t$ where they are well defined, i.e., except at a finite set of time instants $t$.
} 


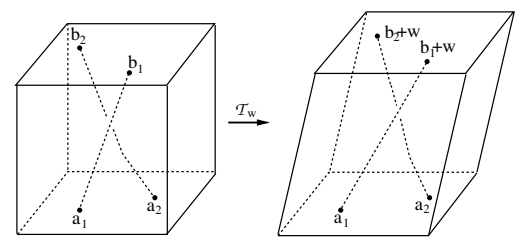

FIG. 3.1. Tilt operation $\mathcal{T}_{w}$ on a 2-maneuver.

Remark 1 (Geodesics in a manifold with boundary). Problem (3.4) can be formulated in an alternative way. By viewing $\alpha=\left(\alpha_{1}, \cdots, \alpha_{n}\right) \in \mathbf{P}(\mathbf{a}, \mathbf{b})$ as a curve in $\mathbb{R}^{2 n}$, and $\mathbf{a}, \mathbf{b}$ as two points in $\mathbb{R}^{2 n}$, a conflict-free maneuver in $\mathbf{P}(R, \mathbf{a}, \mathbf{b})$ corresponds to a curve in $\mathbb{R}^{2 n}$ joining $\mathbf{a}$ to $\mathbf{b}$ and avoiding the obstacle $W$ defined by

$$
W=\left\{\left(p_{1}, \cdots, p_{n}\right) \in \mathbb{R}^{2 n}: p_{i} \in \mathbb{R}^{2}, 1 \leq i \leq n \text {, and }\left\|p_{j}-p_{k}\right\|<R \text { for some } j \neq k\right\} .
$$

If the coefficients $\mu_{i}, i=1, \ldots, n$, are identical, then the $\mu$-energy of a joint maneuver is proportional to the energy of the corresponding curve in $\mathbb{R}^{2 n}$. Therefore, problem (3.4) is equivalent to finding the curve in $\mathbb{R}^{2 n} \backslash W$ joining $\mathbf{a}$ to $\mathbf{b}$ with the least energy, which is a minimizing geodesic of $\mathbb{R}^{2 n} \backslash W$ connecting $\mathbf{a}$ to $\mathbf{b}$. Note that $\mathbb{R}^{2 n} \backslash W$ is a manifold with nonsmooth boundary whose fundamental group is isomorphic to $\mathbf{P} \mathbf{B}_{n}$ by Theorem 2.5. The general case of arbitrary $\left\langle\mu_{i}\right\rangle_{i=1}^{n}$ can be reduced to this special case by scaling the $p_{i}$ axes of $\mathbb{R}^{2 n}$ by a factor of $\sqrt{\mu_{i}}, i=1, \cdots, n$. The interested readers are referred to [17] for further details.

The rest of this section is devoted to the solution of problem (3.4), a variational problem with complicated and nonsmooth constraints. Inspired by the braid representation introduced in Section 2, we propose various transformations of joint maneuvers that preserve the MSE, and use these transformations in the variational analysis to obtain necessary conditions for a maneuver $\alpha^{*} \in \mathbf{P}(R, \mathbf{a}, \mathbf{b})$ to be optimal.

3.2. $\mu$-alignment of optimal conflict-free maneuvers. As explained in Section 2, each conflict-free maneuver $\alpha \in \mathbf{P}(R, \mathbf{a}, \mathbf{b})$ has a natural braid representation $\hat{\alpha} \in \mathbf{P B}(\mathbf{a}, \mathbf{b})$, whose $n$ strings are determined by the images of the maps $t \mapsto\left(\alpha_{i}(t), t\right)$, $t \in T, i=1, \cdots, n$. Furthermore, $\hat{\alpha}$ satisfies the $R$-separation property in that the intersection of $\hat{\alpha}$ with the plane $t=\tau$ for any $\tau \in T$ consists of $n$ points whose pairwise minimum distance is at least $R$. All the operations on conflict-free maneuvers we shall introduce in the following preserve this separation property in the braid representation, hence they are indeed transformations of conflict-free maneuvers.

For each $w \in \mathbb{R}^{2}$, denote by $\mathbf{b}+w$ the $n$-tuple $\left(b_{1}+w, \cdots, b_{n}+w\right)$.

Definition 3.1 (Tilt operator $\left.\mathcal{T}_{w}\right)$. The tilt operator $\mathcal{T}_{w}: \mathbf{P}(R, \mathbf{a}, \mathbf{b}) \rightarrow$ $\mathbf{P}(R, \mathbf{a}, \mathbf{b}+w)$ is a map such that for any $\alpha \in \mathbf{P}(R, \mathbf{a}, \mathbf{b}), \beta=\mathcal{T}_{w}(\alpha)$ is defined by

$$
\beta_{i}(t)=\alpha_{i}(t)+\frac{t-t_{0}}{t_{f}-t_{0}} w, \quad t \in T, \quad i=1, \cdots, n .
$$

It is easily seen that $\mathcal{T}_{w}$ is MSE-preserving in the sense that $\alpha$ and $\mathcal{T}_{w}(\alpha)$ have the same MSE. Hence $\mathcal{T}_{w}$ maps $\mathbf{P}(R, \mathbf{a}, \mathbf{b})$ into $\mathbf{P}(R, \mathbf{a}, \mathbf{b}+w)$. In fact, $\mathcal{T}_{w}$ is a bijection from $\mathbf{P}(R, \mathbf{a}, \mathbf{b})$ to $\mathbf{P}(R, \mathbf{a}, \mathbf{b}+w)$ since $\mathcal{T}_{w} \circ \mathcal{T}_{-w}=\mathcal{T}_{-w} \circ \mathcal{T}_{w}=i d$. In the braid representation, $\hat{\beta}$ is obtained by tilting $\hat{\alpha}$ linearly, hence the name for the operator $\mathcal{T}_{w}$. More precisely, in order to get $\hat{\beta}$ from $\hat{\alpha}$, the plane $t=t_{0}$ is kept invariant (shifted 
by 0 ), the plane $t=t_{f}$ is shifted by $w$, and each intermediate plane $t=\tau, \tau \in\left(t_{0}, t_{f}\right)$, is shifted by an amount determined by the linear interpolation of 0 and $w$ according to the position of $\tau$ in $T$. Fig. 3.1 illustrates the effect of the $\mathcal{T}_{w}$ operator on the braid representation of a 2 -maneuver.

The importance of introducing $\mathcal{T}_{w}$ lies in the following result.

Proposition 3.2. Suppose that $\alpha^{*}$ is a conflict-free maneuver in $\mathbf{P}(R, \mathbf{a}, \mathbf{b})$ with the least $\mu$-energy. Fix $w \in \mathbb{R}^{2}$. Then $\beta^{*}=\mathcal{T}_{w}\left(\alpha^{*}\right)$ is a conflict-free maneuver in $\mathbf{P}(R, \mathbf{a}, \mathbf{b}+w)$ with the least $\mu$-energy.

Proof. For any $\beta \in \mathbf{P}(R, \mathbf{a}, \mathbf{b}+w)$, let $\alpha=\mathcal{T}_{-w}(\beta)$. Then $\alpha \in \mathbf{P}(R, \mathbf{a}, \mathbf{b})$ and $J_{\mu}(\beta)$ can be expressed as

$$
\begin{aligned}
J_{\mu}(\beta) & =\frac{1}{2} \int_{t_{0}}^{t_{f}} \sum_{i=1}^{n} \mu_{i}\left\|\dot{\beta}_{i}(t)\right\|^{2} d t=\frac{1}{2} \int_{t_{0}}^{t_{f}} \sum_{i=1}^{n} \mu_{i}\left\|\dot{\alpha}_{i}(t)+\frac{w}{t_{f}-t_{0}}\right\|^{2} d t \\
& =\frac{1}{2} \int_{t_{0}}^{t_{f}} \sum_{i=1}^{n} \mu_{i}\left\|\dot{\alpha}_{i}(t)\right\|^{2} d t+\int_{t_{0}}^{t_{f}} \frac{w^{T}}{t_{f}-t_{0}} \sum_{i=1}^{n} \mu_{i} \dot{\alpha}_{i}(t) d t+\frac{\|w\|^{2}}{2\left(t_{f}-t_{0}\right)} \\
& =J_{\mu}(\alpha)+\frac{w^{T}\left[\sum_{i=1}^{n} \mu_{i}\left(b_{i}-a_{i}\right)+w / 2\right]}{t_{f}-t_{0}} .
\end{aligned}
$$

Note that the second term in the last equation of (3.5) is a constant independent of $\beta$. Denote it by $C$. It follows by equation (3.5) and the optimality of $\alpha^{*}$ that $J_{\mu}(\beta) \geq J_{\mu}\left(\alpha^{*}\right)+C, \forall \beta \in \mathbf{P}(R, \mathbf{a}, \mathbf{b}+w)$, with the equality if $\alpha=\alpha^{*}$, i.e. $\beta=\beta^{*}$. $\mathbf{\square}$

Consider arbitrary starting and destination positions $\mathbf{a}$ and $\mathbf{b}$, and set $\mathbf{b}^{\prime} \triangleq \mathbf{b}+w$ where $w=\sum_{i=1}^{n} \mu_{i}\left(a_{i}-b_{i}\right)$. Then $\mathbf{a}$ and $\mathbf{b}^{\prime}$ are $\mu$-aligned in the sense that they have the same $\mu$-centroid, i.e.,

$$
\sum_{i=1}^{n} \mu_{i} a_{i}=\sum_{i=1}^{n} \mu_{i} b_{i}^{\prime}
$$

By Proposition 3.2, solutions to problem (3.4) for general $\mathbf{a}$ and $\mathbf{b}$ can be obtained from solutions to problem (3.4) for $\mu$-aligned $\mathbf{a}$ and $\mathbf{b}^{\prime}$ by applying the tilt operator $\mathcal{T}_{-w}$ with $w=\sum_{i=1}^{n} \mu_{i}\left(a_{i}-b_{i}\right)$. This is the reason why we shall focus on the special case of $\mu$-aligned $\mathbf{a}$ and $\mathbf{b}$.

The next transformation we shall introduce is the drift operation. Let $\gamma: T \rightarrow \mathbb{R}^{2}$ be a continuous and piecewise $C^{2}$ map such that $\gamma\left(t_{0}\right)=\gamma\left(t_{f}\right)=0$.

Definition 3.3 (Drift operator $\mathcal{D}_{\gamma}$ ). The drift operator $\mathcal{D}_{\gamma}: \mathbf{P}(R, \mathbf{a}, \mathbf{b}) \rightarrow$ $\mathbf{P}(R, \mathbf{a}, \mathbf{b})$ is a map such that for any $\alpha \in \mathbf{P}(R, \mathbf{a}, \mathbf{b}), \beta=\mathcal{D}_{\gamma}(\alpha)$ is defined by

$$
\beta_{i}(t)=\alpha_{i}(t)+\gamma(t), \quad t \in T, \quad i=1, \cdots, n .
$$

In the braid representation, $\hat{\beta}$ is obtained from $\hat{\alpha}$ by drifting each plane $t=\tau, \tau \in T$, by an offset $\gamma(\tau) \in \mathbb{R}^{2}$. It can be verified that $\mathcal{D}_{\gamma}$ is MSE-preserving and a bijection of $\mathbf{P}(R, \mathbf{a}, \mathbf{b})$ onto itself since $\mathcal{D}_{\gamma} \circ \mathcal{D}_{-\gamma}=\mathcal{D}_{-\gamma} \circ \mathcal{D}_{\gamma}=i d$. By using the drift operator, we can prove the following result.

Proposition 3.4. Suppose that $\mathbf{a}$ and $\mathbf{b}$ are $\mu$-aligned and $\alpha^{*} \in \mathbf{P}(R, \mathbf{a}, \mathbf{b})$ is an optimal solution to problem (3.4). Then

$$
\sum_{i=1}^{n} \mu_{i} \alpha_{i}^{*}(t)=\sum_{i=1}^{n} \mu_{i} a_{i}=\sum_{i=1}^{n} \mu_{i} b_{i}, \quad \forall t \in T .
$$


Proof. For each $\lambda \in \mathbb{R}$ define $\beta_{\lambda} \triangleq \mathcal{D}_{\lambda \gamma}\left(\alpha^{*}\right)$. Note that $\beta_{\lambda} \in \mathbf{P}(R, \mathbf{a}, \mathbf{b})$ and $\beta_{0}=\alpha^{*}$. Moreover,

$$
\begin{aligned}
J_{\mu}\left(\beta_{\lambda}\right) & =\frac{1}{2} \int_{t_{0}}^{t_{f}} \sum_{i=1}^{n} \mu_{i}\left\|\dot{\alpha}_{i}^{*}(t)+\lambda \dot{\gamma}(t)\right\|^{2} d t \\
& =J_{\mu}\left(\alpha^{*}\right)+\frac{\lambda^{2}}{2} \int_{t_{0}}^{t_{f}}\|\dot{\gamma}(t)\|^{2} d t+\lambda \int_{t_{0}}^{t_{f}} \dot{\gamma}(t)^{T} \sum_{i=1}^{n} \mu_{i} \dot{\alpha}_{i}^{*}(t) d t .
\end{aligned}
$$

The difference $J_{\mu}\left(\beta_{\lambda}\right)-J_{\mu}\left(\alpha^{*}\right)$ is a quadratic function of $\lambda$, which, by the optimality of $\alpha^{*}$, must be nonnegative for all $\lambda$. Hence we have $\int_{t_{0}}^{t_{f}} \dot{\gamma}(t)^{T} \sum_{i=1}^{n} \mu_{i} \dot{\alpha}_{i}^{*}(t) d t=0$, which must hold for any choice of $\gamma$ such that $\gamma\left(t_{0}\right)=\gamma\left(t_{f}\right)=0$. Since $\mathbf{a}$ and $\mathbf{b}$ are $\mu$-aligned, we can choose $\gamma(t)=\sum_{i=1}^{n} \mu_{i} \alpha_{i}^{*}(t)-\sum_{i=1}^{n} \mu_{i} a_{i}$. Given that $\alpha^{*}$ is piecewise $C^{2}$, this leads to $\sum_{i=1}^{n} \mu_{i} \dot{\alpha}_{i}^{*}(t)=0$ for almost all $t \in T$, and hence, by integration, to the desired conclusion. $\square$

We can now use Proposition 3.2 to get the formulation of Proposition 3.4 for arbitrary $\mathbf{a}$ and $\mathbf{b}$.

Corollary 3.5. Suppose that $\alpha^{*} \in \mathbf{P}(R, \mathbf{a}, \mathbf{b})$ is an optimal solution to problem (3.4). Then

$$
\sum_{i=1}^{n} \mu_{i} \alpha_{i}^{*}(t)=\sum_{i=1}^{n} \mu_{i} a_{i}+\frac{t-t_{0}}{t_{f}-t_{0}}\left(\sum_{i=1}^{n} \mu_{i} b_{i}-\sum_{i=1}^{n} \mu_{i} a_{i}\right), \quad \forall t \in T .
$$

In other words, the $\mu$-centroid of $\left\langle\alpha_{i}^{*}(t)\right\rangle_{i=1}^{n}$ moves from the $\mu$-centroid of a at time $t_{0}$ to the $\mu$-centroid of $\mathbf{b}$ at time $t_{f}$ with constant velocity.

Remark 2. The results in Proposition 3.2, Proposition 3.4 and Corollary 3.5 for conflict-free maneuvers in $\mathbb{R}^{2}$ are still valid when the underlying space is $\mathbb{R}^{k}$ with $k>2$. These can be proved by following exactly the same procedure as in the $\mathbb{R}^{2}$ case.

REMARK 3. A geometric interpretation of Corollary 3.5 can be given in the case when the $\mu_{i}$ 's are identical. Let $W$ be the obstacle in $\mathbb{R}^{2 n}$ defined as in Remark 1. An important observation is that $W$ is cylindrical in the direction of the 2-dimensional subspace $N$ spanned by vectors $(1,0,1,0, \cdots, 1,0)^{T}$ and $(0,1,0,1, \cdots, 0,1)^{T}$ in $\mathbb{R}^{2 n}$, in the sense that for any $x \in \mathbb{R}^{2 n}, x \in W$ if and only if $x+N \subset W$. Let $V$ be the orthogonal complement of $N$ in $\mathbb{R}^{2 n}$. Then $\mathbf{a}$ and $\mathbf{b}$ are $\mu$-aligned if and only if $\mathbf{a}$ and $\mathbf{b}$ are on the same $V$-slice in $\mathbb{R}^{2 n}$, i.e., if and only if $\mathbf{a}-\mathbf{b} \in V$. The conclusions of Proposition 3.2 and Corollary 3.5 say that for $\mathbf{a}$ and $\mathbf{b}$ that are not necessarily $\mu$ aligned, the shortest geodesic in $\mathbb{R}^{2 n} \backslash W$ from $\mathbf{a}$ to $\mathbf{b}$ can be decomposed into two parts: its projection onto $N$, which is a constant speed motion along the straight line from $\pi_{N}(\mathbf{a})$ to $\pi_{N}(\mathbf{b})$, where $\pi_{N}: \mathbb{R}^{2 n} \rightarrow N$ denotes the orthogonal projection map onto $N$; and its projection onto $V$, which is the shortest geodesic in $V \cap W^{c}$ connecting $\pi_{V}(\mathbf{a})$ and $\pi_{V}(\mathbf{b})$, where $\pi_{V}: \mathbb{R}^{2 n} \rightarrow V$ denotes the orthogonal projection map onto $V$. Since $V$ is of dimension $2 n-2$, this effectively reduces the dimension of the problem by 2 .

3.3. Optimal conflict-free maneuvers for two agents. We now show that the solution to problem (3.4) in the case when there are only two agents follows directly from Corollary 3.5 .

Assume that $\mathbf{a}=\left(a_{1}, a_{2}\right)$ and $\mathbf{b}=\left(b_{1}, b_{2}\right)$ are $\mu$-aligned, and denote by $c$ their common $\mu$-centroid. If $\alpha^{*}=\left(\alpha_{1}^{*}, \alpha_{2}^{*}\right) \in \mathbf{P}(R, \mathbf{a}, \mathbf{b})$ is an optimal solution to problem (3.4), then, by Proposition 3.4, the $\mu$-centroid of $\alpha_{1}^{*}(t)$ and $\alpha_{2}^{*}(t)$ is equal to $c$ for any 

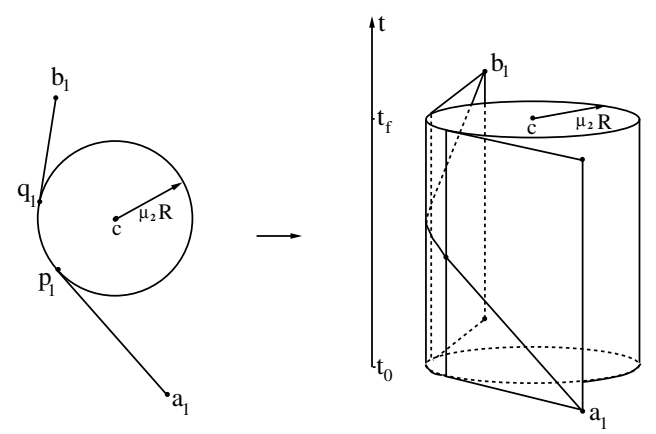

FIG. 3.2. Optimal 2-maneuver and its braid representation.

$t \in T$, or equivalently,

$$
\alpha_{1}^{*}(t)-c=-\frac{\mu_{2}}{\mu_{1}}\left(\alpha_{2}^{*}(t)-c\right), \quad \forall t \in T .
$$

From equation (3.7), it then follows that the energies of $\alpha_{1}^{*}$ and $\alpha_{2}^{*}$ are related by $\mu_{1}^{2} J\left(\alpha_{1}^{*}\right)=\mu_{2}^{2} J\left(\alpha_{2}^{*}\right)$, and that the separation constraint $\left\|\alpha_{1}^{*}(t)-\alpha_{2}^{*}(t)\right\| \geq R$ is equivalent to $\left\|\alpha_{1}^{*}(t)-c\right\| \geq \mu_{2} R$. Therefore, problem (3.4) can be reduced to

$$
\text { minimize } J\left(\alpha_{1}\right) \text { subject to } \alpha_{1} \in \mathbf{P}_{1} \text { and } \alpha_{1}: T \rightarrow B^{c}\left(c, \mu_{2} R\right) \text {, }
$$

where $B^{c}\left(c, \mu_{2} R\right)$ denotes the complement in $\mathbb{R}^{2}$ of the open disk of radius $\mu_{2} R$ centered at $c$. Thus the problem becomes finding the minimum energy maneuver for a single agent in the presence of the static obstacle $B\left(c, \mu_{2} R\right)$.

By assumption, both $a_{1}$ and $b_{1}$ belong to $B^{c}\left(c, \mu_{2} R\right)$ since otherwise the problem is infeasible. From the discussion at the beginning of Section 3.1, we know that the optimal solution to problem (3.8) is a constant speed motion along the shortest curve joining $a_{1}$ to $b_{1}$ while avoiding the obstacle $B\left(c, \mu_{2} R\right)$. Let $\partial B$ be the boundary of the disk $B\left(c, \mu_{2} R\right)$. The geometric construction of the shortest curve within a given fundamental type is shown in Fig. 3.2. The curve is composed of three pieces: first from $a_{1}$ to $p_{1} \in \partial B$ along a straight line tangent to $\partial B$, then from $p_{1}$ to $q_{1}$ along $\partial B$, and finally from $q_{1}$ to $b_{1}$ along another straight line tangent to $\partial B$. Here choosing a fundamental type is equivalent to choosing a side of the cylinder in the braid representation. The globally optimal solution $\alpha_{1}^{*}$ is the one of the two locally optimal solutions with shorter arc length (or any one of them if they have the same length). $\alpha_{2}^{*}$ is then obtained from $\alpha_{1}^{*}$ by equation (3.7). This is for the $\mu$-aligned case. Denote by $\gamma_{i}^{*}(\mathbf{a}, \mathbf{b}), i=1,2$, the obtained optimal maneuvers. For the general case when $\mathbf{a}$ and $\mathbf{b}$ are not necessarily $\mu$-aligned, we have by Proposition 3.2

Theorem 3.6 (Optimal conflict-free 2-maneuver). If $n=2$, then the optimal solution $\alpha^{*} \in \mathbf{P}(R, \mathbf{a}, \mathbf{b})$ to problem (3.4) is given by:

$$
\left\{\begin{array}{l}
\alpha_{1}^{*}(t)=\gamma_{1}^{*}(\mathbf{a}, \mathbf{b}+w)(t)-\frac{t-t_{0}}{t_{f}-t_{0}} w \\
\alpha_{2}^{*}(t)=\gamma_{2}^{*}(\mathbf{a}, \mathbf{b}+w)(t)-\frac{t-t_{0}}{t_{f}-t_{0}} w
\end{array}, \quad \forall t \in T,\right.
$$

where $w=\mu_{1} a_{1}-\mu_{1} b_{1}+\mu_{2} a_{2}-\mu_{2} b_{2}$.

Consider the case when the priority of agent 1 is much higher than that of agent 2 , which can be modeled by $\mu_{2} \simeq 0$. In the $\mu$-aligned case, this implies $a_{1} \simeq b_{1} \simeq c$, 

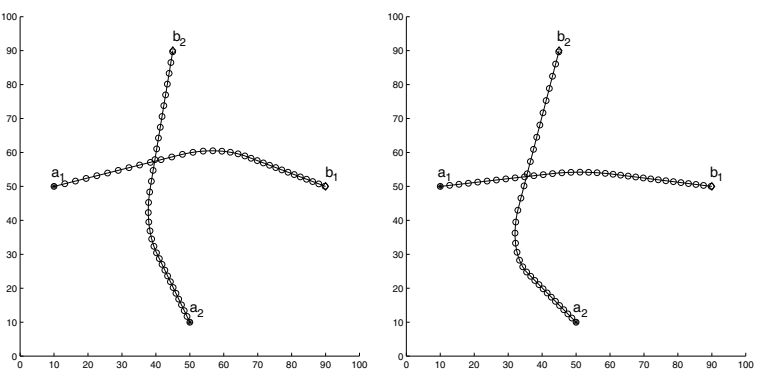

FIG. 3.3. Optimal 2-maneuvers $(R=30)$. Left: $\mu_{1}=\mu_{2}=0.5$, Right: $\mu_{1}=0.8, \mu_{2}=0.2$.

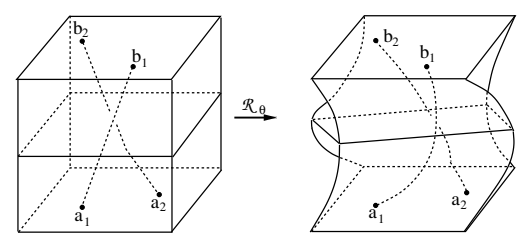

FIG. 3.4. Twist operation $\mathcal{R}_{\theta}$ on a 2-maneuver.

and that the radius of the disk $B\left(c, \mu_{2} R\right)$ is about 0 . Therefore, $\gamma_{1}^{*}$ is nearly a zero motion. For general $\mathbf{a}$ and $\mathbf{b}$, it follows from Theorem 3.6 that the optimal maneuver for agent 1 is almost a constant speed motion along the line segment from $a_{1}$ to $b_{1}$. Hence, as expected, agent 2 is the one assuming most of the responsibility of avoiding conflicts.

Shown in Fig. 3.3 are the plots of optimal conflict-free maneuvers for a typical 2agent encounter with two different sets of priorities. The circles represent the positions of the two agents at evenly distributed time instants. The plots show that, in the case when $\mathbf{a}$ and $\mathbf{b}$ are not $\mu$-aligned, the speeds of the agents in the optimal maneuvers are not constant. As the priority of agent 1 increases, however, its optimal maneuver gets closer to the constant speed motion along the straight line connecting $a_{1}$ to $b_{1}$.

3.4. Twist optimality. Another MSE-preserving operator can be introduced as follows. Suppose that $\theta: T \rightarrow \mathbb{R}$ is a continuous and piecewise $C^{2}$ map satisfying $\theta\left(t_{0}\right)=0, \theta\left(t_{f}\right)=2 k \pi$ for some $k \in \mathbb{Z}$.

Definition 3.7 (Twist operator $\mathcal{R}_{\theta}$ ). The twist operator $\mathcal{R}_{\theta}: \mathbf{P}(R, \mathbf{a}, \mathbf{b}) \rightarrow$ $\mathbf{P}(R, \mathbf{a}, \mathbf{b})$ is a map such that for any $\alpha \in \mathbf{P}(R, \mathbf{a}, \mathbf{b}), \beta=\mathcal{R}_{\theta}(\alpha)$ is defined by

$$
\beta_{i}(t)=T_{\theta(t)} \alpha_{i}(t), \quad t \in T, \quad i=1, \cdots, n,
$$

where $T_{\theta(t)}$ is the matrix corresponding to a rotation of $\theta(t)$ counterclockwise:

$$
T_{\theta(t)}=\left(\begin{array}{cc}
\cos [\theta(t)] & -\sin [\theta(t)] \\
\sin [\theta(t)] & \cos [\theta(t)]
\end{array}\right) .
$$

The constraints on $\theta\left(t_{0}\right)$ and $\theta\left(t_{f}\right)$ ensure that $\mathcal{R}_{\theta}(\alpha)$ and $\alpha$ have the same starting and ending positions. It is easy to see that $\mathcal{R}_{\theta}$ is MSE-preserving and hence has its image in $\mathbf{P}(R, \mathbf{a}, \mathbf{b})$. Fig. 3.4 shows the effect of $\mathcal{R}_{\theta}(k=0)$ on the braid representation of a 2-maneuver, which motivates the name "twist operator" for it.

By considering the perturbed maneuvers generated by $\mathcal{R}_{\theta}$, we have 
Proposition 3.8. Suppose that $\alpha^{*} \in \mathbf{P}(R, \mathbf{a}, \mathbf{b})$ is an optimal solution to problem (3.4). Fix $s \in \mathbb{R}^{2}$. Then

$$
\frac{1}{2} \sum_{i=1}^{n} \mu_{i}\left(\alpha_{i}^{*}(t)-s\right)^{T} T_{-\frac{\pi}{2}} \dot{\alpha}_{i}^{*}(t)=C, \quad \forall t \in T,
$$

where $C$ is a constant belonging to $\left[-\frac{\pi}{z}, \frac{\pi}{z}\right]$, with $z \triangleq 2 \int_{t_{0}}^{t_{f}}\left[\sum_{i=1}^{n} \mu_{i}\left\|\alpha_{i}^{*}(t)-s\right\|^{2}\right]^{-1} d t$.

Proof. See Appendix A. $\square$

If $k \neq 0$, then the operator $\mathcal{R}_{\theta}$ changes the homotopy type of conflict-free maneuvers in $\mathbf{P}(R, \mathbf{a}, \mathbf{b})$, thus enabling us to compare the performance of conflict-free maneuvers of different types. In this sense, the result in Proposition 3.8 is global. We illustrate this statement by the following example.

EXAMPle 1. Assume that $n=2$ and $\mu_{1}=\mu_{2}=\frac{1}{2}$. Let $t_{0}=0$ and $t_{f}=\tau$ for some $\tau \in(0,2 \pi)$. Set $a_{1}=\frac{R}{2}(1,0)^{T}, b_{1}=\frac{R}{2}(\cos \tau, \sin \tau)^{T}, a_{2}=-a_{1}$ and $b_{2}=-b_{1}$. Consider the conflict-free maneuvers $\alpha$ and $\beta$ in $\mathbf{P}(R, \mathbf{a}, \mathbf{b})$ defined by $\alpha_{1}(t)=\frac{R}{2}(\cos t, \sin t)^{T}, \alpha_{2}(t)=-\alpha_{1}(t)$, and $\beta_{1}(t)=\frac{R}{2}\left(\cos \left(\frac{\tau-2 \pi}{\tau} t\right), \sin \left(\frac{\tau-2 \pi}{\tau} t\right)\right)^{T}$, $\beta_{2}(t)=-\beta_{1}(t)$, for all $t \in[0, \tau]$. The two agents under maneuver $\alpha(\beta)$ rotate around the origin at constant angular velocity counterclockwise (clockwise) during $[0, \tau]$. Note that $\beta$ can be obtained from $\alpha$ by applying the twist operator $\mathcal{R}_{\theta}$ with $\theta(t)=-2 \pi t / \tau$ satisfying $\theta(\tau)=-2 \pi$, and that $\alpha$ and $\beta$ belong to different types. Since $\mathbf{a}$ and $\mathbf{b}$ are $\mu$-aligned, the results in Section 3.3 imply that $\alpha$ and $\beta$ are the optimal solutions to problem (3.4) restricted to the two fundamental types. The global optimal solution is the one of them with smaller arc length, which can be easily seen to be $\alpha$ if $\tau \in(0, \pi)$ and $\beta$ if $\tau \in(\pi, 2 \pi)$. This conclusion can also be reached directly by an application of Proposition 3.8. In fact, if we choose $s=0$ and compute $C$ and $z$ defined in Proposition 3.8 with $\alpha$ in the place of $\alpha^{*}$, we get $C=R^{2} / 8$ and $z=8 \tau / R^{2}$, and the inequality $|C| \leq \pi / z$ becomes $\tau \leq \pi$, which implies that $\alpha$ is not globally optimal for $\tau \in(\pi, 2 \pi)$. If we compute $C$ and $z$ with $\beta$ in the place of $\alpha^{*}$, we get $C=R^{2}(\tau-2 \pi) / 8 \tau$ and $z=8 \tau / R^{2}$, and the inequality $|C| \leq \pi / z$ becomes $\tau \geq \pi$. Hence $\beta$ is not globally optimal for $\tau \in(0, \pi)$.

Note that by choosing different $s \in \mathbb{R}^{2}$, Proposition 3.8 provides a family of inequalities of the form $-\frac{\pi}{z} \leq C \leq \frac{\pi}{z}$ that an optimal solution $\alpha^{*}$ to problem (3.4) must satisfy, where $C$ and $z$ are functions of $s$ and $\alpha^{*}$. In the case when $\mathbf{a}$ and $\mathbf{b}$ are $\mu$-aligned, by Proposition 3.4, we have $\sum_{i=1}^{n} \mu_{i} s^{T} T_{-\frac{\pi}{2}} \dot{\alpha}_{i}^{*}(t) \equiv 0$. Hence the inequality becomes

$$
\left|\frac{1}{2} \sum_{i=1}^{n} \mu_{i} \alpha_{i}^{*}(t)^{T} T_{-\frac{\pi}{2}} \dot{\alpha}_{i}^{*}(t)\right| \leq \frac{\pi}{2}\left\{\int_{t_{0}}^{t_{f}}\left[\sum_{i=1}^{n} \mu_{i}\left\|\alpha_{i}^{*}(t)-s\right\|^{2}\right]^{-1} d t\right\}^{-1} .
$$

The most restrictive bound is obtained by setting $s$ equal to the common $\mu$-centroid of $\mathbf{a}$ and $\mathbf{b}$, which minimizes the right-hand-side of the above equation. Moreover, one can derive further optimality conditions by applying Proposition 3.8 to $\mathcal{T}_{w}\left(\alpha^{*}\right)$ for any $w \in \mathbb{R}^{2}$, since by Proposition $3.2 \mathcal{T}_{w}\left(\alpha^{*}\right)$ is optimal in $\mathbf{P}(R, \mathbf{a}, \mathbf{b}+w)$.

3.5. Analysis by partial operators. Further optimality conditions can be derived by considering those transformations that change the maneuvers of only a subset of the $n$ agents (partial operators).

Let $\alpha$ be an arbitrary conflict-free maneuver in $\mathbf{P}(R, \mathbf{a}, \mathbf{b})$. At each time $t \in T$, we can construct an undirected graph $G_{\alpha}(t)$ as follows: $G_{\alpha}(t)$ has $n$ vertices, numbered from 1 to $n$, corresponding to the $n$ agents, and an edge connects vertices $i$ and $j$ if 


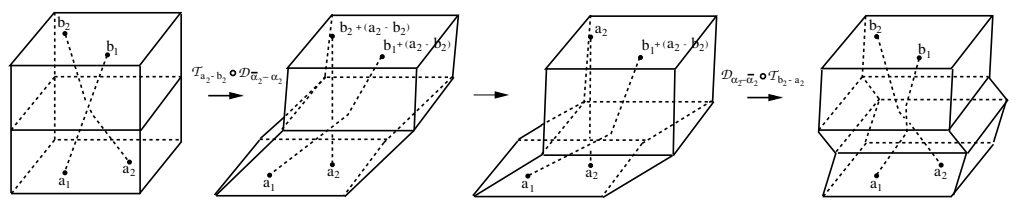

FIG. 3.5. Slide operation $\mathcal{L}_{h}^{12}$ on braids.

and only if $\left\|\alpha_{i}(t)-\alpha_{j}(t)\right\|=R$. If there does exist an edge between vertex $i$ and vertex $j$ in $G_{\alpha}(t)$, we say that agent $i$ and agent $j$ contact at time $t . G_{\alpha}(t)$ is then called the contact graph of $\alpha$ at time $t$.

We start from a very special case. Assume that $\alpha$ is a conflict-free maneuver in $\mathbf{P}(R, \mathbf{a}, \mathbf{b})$ such that during the whole encounter the distance of agent 1 from any of the other agents is strictly greater than $R$ except possibly from agent 2 , i.e., $\left\|\alpha_{1}(t)-\alpha_{i}(t)\right\|>R, \forall t \in T, i=3, \cdots, n$. We shall introduce operators that leave $\alpha_{i}$ unchanged for $i=2,3, \cdots, n$ and perturb $\alpha_{1}$ slightly, so that the perturbed $\alpha_{1}$ has the same minimum distance from $\alpha_{2}$ in the time interval $T$. If such a perturbation is small enough, then the perturbed $\alpha_{1}$ does not cause a conflict between agent 1 and any of the agents with index $i \geq 3$, given that their original minimum distance in the time interval $T$ was strictly greater than $R$.

Let $h: T \rightarrow T$ be a reparameterization of $T$, i.e., a bijection such that both $h$ and $h^{-1}$ are continuous and piecewise $C^{2}$, and $h\left(t_{0}\right)=t_{0}$ and $h\left(t_{f}\right)=t_{f}$.

Definition 3.9 (Partial slide operator $\mathcal{L}_{h}^{12}$ ). The partial slide operator $\mathcal{L}_{h}^{12}$ : $\mathbf{P}(R, \mathbf{a}, \mathbf{b}) \rightarrow \mathbf{P}(\mathbf{a}, \mathbf{b})$ is a map such that for any $\alpha \in \mathbf{P}(R, \mathbf{a}, \mathbf{b}), \beta=\mathcal{L}_{h}^{12}(\alpha)$ is defined by:

$$
\left\{\begin{array}{l}
\beta_{1}(t)=\alpha_{1}[h(t)]-\alpha_{2}[h(t)]+\alpha_{2}(t), \quad t \in T, \\
\beta_{i}(t)=\alpha_{i}(t), \quad t \in T, \quad i=2, \cdots, n .
\end{array}\right.
$$

Note that $\inf _{t \in T}\left\|\beta_{1}(t)-\beta_{2}(t)\right\|=\inf _{t \in T}\left\|\alpha_{1}(t)-\alpha_{2}(t)\right\|$, and that for $h$ sufficiently close to the identity map, the minimum distance in the time interval $T$ between $\beta_{1}$ and $\beta_{i}$ is greater than $R$ for $i \geq 3$ by our assumption on $\alpha$. These two conditions together imply that $\beta \in \mathbf{P}(R, \mathbf{a}, \mathbf{b})$.

Fig. 3.5 shows how $\beta$ is constructed geometrically. First, the operator $\mathcal{D}_{\bar{\alpha}_{2}-\alpha_{2}}$ is performed on $\left(\alpha_{1}, \alpha_{2}\right)$ to "straighten" the string corresponding to $\alpha_{2}$, where $\bar{\alpha}_{2}$ denotes the constant velocity motion along the straight line between $a_{2}$ and $b_{2}$. Next, the operator $\mathcal{T}_{a_{2}-b_{2}}$ is applied to the resulting 2-maneuver to get a 2-maneuver $\gamma=\left(\gamma_{1}, \gamma_{2}\right)$ with $\gamma_{1}=\alpha_{1}-\alpha_{2}+a_{2}$ and $\gamma_{2} \equiv a_{2}$. Then, $\gamma$ is reparameterized by $h$ to obtain $\eta=\left(\eta_{1}, \eta_{2}\right)$ with $\eta_{1}=\left(\alpha_{1} \circ h\right)-\left(\alpha_{2} \circ h\right)+a_{2}$ and $\eta_{2} \equiv a_{2}$. Finally, the reverse procedures of the second and first steps are applied subsequently to obtain $\left(\beta_{1}, \beta_{2}\right)$ from $\eta$. Roughly speaking, $\hat{\beta}$ is obtained by "sliding" $\hat{\alpha}_{1}$ along $\hat{\alpha}_{2}$, hence the name "slide operator" for $\mathcal{L}_{h}^{12}$. Note that the superscript and the subscript in $\mathcal{L}_{h}^{12}$ indicate respectively the two strings the operator works on and the reparameterization used.

By using the partial slide operator to generate the perturbation in the variational analysis, we get (see [12] for the detailed proof):

Proposition 3.10. Suppose that $\alpha^{*} \in \mathbf{P}(R, \mathbf{a}, \mathbf{b})$ is an optimal solution to problem (3.4), and that there exists a subinterval $\left(t_{0}^{\prime}, t_{f}^{\prime}\right) \subset T$ such that $\left\|\alpha_{1}^{*}(t)-\alpha_{i}^{*}(t)\right\|>R$, $i=3, \cdots, n$, for all $t \in\left(t_{0}^{\prime}, t_{f}^{\prime}\right)$. Then $\alpha^{*}$ satisfies

$$
\ddot{\alpha}_{1}^{*}(t)^{T}\left(\dot{\alpha}_{1}^{*}(t)-\dot{\alpha}_{2}^{*}(t)\right) \equiv 0, \quad \forall t \in\left(t_{0}^{\prime}, t_{f}^{\prime}\right) .
$$


Instead of sliding $\alpha_{1}$ along $\alpha_{2}$, we can rotate it. Let $\theta: T \rightarrow \mathbb{R}$ be a continuous and piecewise $C^{2}$ map with $\theta\left(t_{0}\right)=\theta\left(t_{f}\right)=0$.

Definition 3.11 (Partial rotation operator $\mathcal{R}_{\theta}^{12}$ ). The partial rotation operator $\mathcal{R}_{\theta}^{12}: \mathbf{P}(R, \mathbf{a}, \mathbf{b}) \rightarrow \mathbf{P}(\mathbf{a}, \mathbf{b})$ is a map such that for any $\alpha \in \mathbf{P}(R, \mathbf{a}, \mathbf{b}), \beta=\mathcal{R}_{\theta}^{12}(\alpha)$ is defined by:

$$
\left\{\begin{array}{l}
\beta_{1}(t)=T_{\theta}(t)\left[\alpha_{1}(t)-\alpha_{2}(t)\right]+\alpha_{2}(t), \quad t \in T \\
\beta_{i}(t)=\alpha_{i}(t), \quad t \in T, \quad i=2, \cdots, n
\end{array}\right.
$$

In the braid representation, $\hat{\beta}$ is obtained by rotating the string $\hat{\alpha}_{1}$ around the string $\hat{\alpha}_{2}$. If $\theta$ is close enough to the zero map, $\beta=\mathcal{R}_{\theta}^{12}(\alpha) \in \mathbf{P}(R, \mathbf{a}, \mathbf{b})$. Similarly to the proof of Proposition 3.10, by using the partial rotation operator, we get ([12]):

Proposition 3.12. Under the hypotheses of Proposition 3.10, $\alpha^{*}$ satisfies

$$
\ddot{\alpha}_{1}^{*}(t)^{T} T_{\frac{\pi}{2}}\left(\alpha_{1}^{*}(t)-\alpha_{2}^{*}(t)\right) \equiv 0, \quad \forall t \in\left(t_{0}^{\prime}, t_{f}^{\prime}\right)
$$

It can be verified that the optimal solution for the two agents case obtained in Theorem 3.6 indeed satisfies both conditions (3.12) and (3.13). Moreover, if one of the two agents has a predetermined maneuver throughout $T$, equations (3.12) and (3.13) will govern the motion of the other agent. Note also that if in addition $\left\|\alpha_{1}^{*}-\alpha_{2}^{*}\right\|=R$ on $\left(t_{0}^{\prime}, t_{f}^{\prime}\right)$, then these two equations are equivalent, since in this case $\left\|\alpha_{1}^{*}-\alpha_{2}^{*}\right\|^{2} \equiv R^{2}$ implies that $\left(\dot{\alpha}_{1}^{*}-\dot{\alpha}_{2}^{*}\right)^{T}\left(\alpha_{1}^{*}-\alpha_{2}^{*}\right) \equiv 0$, i.e., $\dot{\alpha}_{1}^{*}-\dot{\alpha}_{2}^{*}$ and $T_{\frac{\pi}{2}}\left(\alpha_{1}^{*}-\alpha_{2}^{*}\right)$ have the same direction. The intuitive understanding is that, in the braid representation, the slide and rotation operations of a string on the surface of a cylinder lead to the same orthogonal perturbation.

The above idea can be carried out even further. Suppose that the contact graph of an optimal maneuver $\alpha^{*} \in \mathbf{P}(R, \mathbf{a}, \mathbf{b})$ remains constant on some subinterval $\left(t_{0}^{\prime}, t_{f}^{\prime}\right) \subset$ $T$. We can perturb $\alpha^{*}$ by sliding (rotating) slightly the maneuvers of a subset of the $n$ agents with respect to that of agent $i$ in the time subinterval $\left(t_{0}^{\prime}, t_{f}^{\prime}\right)$. To ensure that the perturbed joint maneuver belongs to $\mathbf{P}(R, \mathbf{a}, \mathbf{b})$, any agent in this subset should have a minimum distance strictly greater than $R$ from any of the agents not belonging to the subset, except possibly from agent $i$, in the time interval $\left(t_{0}^{\prime}, t_{f}^{\prime}\right)$. Since $\alpha^{*}$ is optimal, its $\mu$-energy cannot be decreased by such a perturbation. By using the same arguments leading to Proposition 3.10 and Proposition 3.12, we then have ([12])

Proposition 3.13. Suppose that $\alpha^{*} \in \mathbf{P}(R, \mathbf{a}, \mathbf{b})$ is an optimal solution to problem (3.4), and that its contact graph remains constant on some subinterval $\left(t_{0}^{\prime}, t_{f}^{\prime}\right) \subset$ T. Pick any agent, say, agent $i$, and let $\mathcal{I} \subset\{1,2, \cdots, n\} \backslash\{i\}$ be a subset of the remaining agents that corresponds to a maximal connected component of the graph obtained by removing node $i$ and all the edges connected with it from the contact graph during $\left(t_{0}^{\prime}, t_{f}^{\prime}\right)$. Then for all $t \in\left(t_{0}^{\prime}, t_{f}^{\prime}\right)$,

$$
\begin{aligned}
& \sum_{j \in \mathcal{I}} \mu_{j} \ddot{\alpha}_{j}^{*}(t)^{T}\left(\dot{\alpha}_{j}^{*}(t)-\dot{\alpha}_{i}^{*}(t)\right) \equiv 0 \\
& \sum_{j \in \mathcal{I}} \mu_{j} \ddot{\alpha}_{j}^{*}(t)^{T} T_{\frac{\pi}{2}}\left(\alpha_{j}^{*}(t)-\alpha_{i}^{*}(t)\right) \equiv 0 .
\end{aligned}
$$

Note that (3.12) and (3.13) are special cases of (3.14) when $i=2$ and $\mathcal{I}=\{1\}$. 
Proposition 3.13 is the most comprehensive optimality condition we have obtained so far. Next, in Section 3.6 we will show by a simple example how it can be used (together with the global optimality conditions) to determine the optimal maneuver with a particular contact graph. This example will also serve as a counterexample to the conjecture that for each multi-agent encounter, there is a unique optimal conflictfree maneuver within each homotopy type, which is true for $n=2$ by Theorem 3.6.

REMARK 4. All the optimality conditions we have obtained so far admit mechanical interpretations, as will be shown in Section 3.8. However, it should be pointed out that in general they cannot completely characterize the optimal maneuver with an arbitrary contact graph. A complete set of local optimality conditions can be derived by considering all possible local perturbations of maneuvers that preserve the contact graph, or in the light of Remark 1, by writing down the geodesics equation in a suitable Riemannian manifold.

3.6. An interesting example. Consider three agents with equal priorities $\mu_{1}=$ $\mu_{2}=\mu_{3}=\frac{1}{3}$ and $R=1$. Suppose that $\alpha^{*}$ is an optimal conflict-free maneuver for some starting position $\mathbf{a}=\left(a_{1}, a_{2}, a_{3}\right)$ and destination position $\mathbf{b}=\left(b_{1}, b_{2}, b_{3}\right)$ that are $\mu$-aligned with common $\mu$-centroid at the origin, and that on some subinterval of $T$ (which we may assume without loss of generality to be $T$ itself), its contact graph $G_{\alpha^{*}}(t)$ is constant with edges between vertices 1 and 3 and between vertices 2 and 3, but no edges between vertices 1 and 2. Then, by Corollary 3.5 and Proposition 3.13, $\alpha^{*}=\left(\alpha_{1}^{*}, \alpha_{2}^{*}, \alpha_{3}^{*}\right)$ must satisfy for $t \in T$

$$
\left\{\begin{array}{l}
\sum_{i=1}^{3} \alpha_{i}^{*}(t)=0 \\
\ddot{\alpha}_{1}^{*}(t)^{T} T_{\frac{\pi}{2}}\left(\alpha_{1}^{*}(t)-\alpha_{3}^{*}(t)\right)=0 \\
\ddot{\alpha}_{2}^{*}(t)^{T} T_{\frac{\pi}{2}}\left(\alpha_{2}^{*}(t)-\alpha_{3}^{*}(t)\right)=0 \\
\left\|\alpha_{1}^{*}(t)-\alpha_{3}^{*}(t)\right\|=\left\|\alpha_{2}^{*}(t)-\alpha_{3}^{*}(t)\right\|=1
\end{array}\right.
$$

We now show that equation (3.15) is equivalent to the geodesics equation of a suitable Riemannian manifold (a differential manifold together with a smoothly varying positive definite quadratic form on its tangent bundle [8]). Hence, for any set of initial conditions $\alpha_{i}^{*}\left(t_{0}\right), \dot{\alpha}_{i}^{*}\left(t_{0}\right), i=1,2,3$, it has a unique solution for $t$ belonging to a neighborhood of $t_{0}$. First, notice that $\alpha^{*}$ as a curve in $\mathbb{R}^{6}$ lies in the submanifold $Q$ of $\mathbb{R}^{6}$ determined by the first and the last equations of (3.15), namely the set of all those points $\left(x_{1}, y_{1}, x_{2}, y_{2}, x_{3}, y_{3}\right)$ in $\mathbb{R}^{6}$ such that $\sum_{i=1}^{3} x_{i}=\sum_{i=1}^{3} y_{i}=0$, $\left(x_{1}-x_{3}\right)^{2}+\left(y_{1}-y_{3}\right)^{2}=1$ and $\left(x_{2}-x_{3}\right)^{2}+\left(y_{2}-y_{3}\right)^{2}=1 . Q$ is a compact twodimensional submanifold of $\mathbb{R}^{6}$ and admits a global coordinate $\left(\theta_{1}, \theta_{2}\right)$ defined by

$$
\theta_{1}=\arctan \frac{y_{1}-y_{3}}{x_{1}-x_{3}}, \quad \theta_{2}=\arctan \frac{y_{2}-y_{3}}{x_{2}-x_{3}}
$$

$\left(\theta_{1}, \theta_{2}\right)$ takes values in the rectangle $[0,2 \pi] \times[0,2 \pi]$ with opposite edges identified, i.e. the 2 -torus $\mathbb{T}^{2}$. In order to satisfy our assumption that the distance between agent 1 and agent 2 is greater than $R$ during $T, \alpha^{*}$ must lie in an open subset $Q_{0}$ of $Q$ consisting of all those points $\left(x_{1}, y_{1}, x_{2}, y_{2}, x_{3}, y_{3}\right)$ in $Q$ such that $\left(x_{1}-x_{2}\right)^{2}+$ $\left(y_{1}-y_{2}\right)^{2}>1$. In the $\left(\theta_{1}, \theta_{2}\right)$ coordinate, $Q_{0}$ corresponds to an open subset $\mathbb{T}_{0}^{2}$ of $\mathbb{T}^{2}$ obtained by removing from $\mathbb{T}^{2}$ the shaded region shown in Fig. 3.6. Hence topologically $Q_{0}$ is homeomorphic to $S^{1} \times(0,1)$, an untwisted ribbon whose boundary consists of two disjoint circles. 


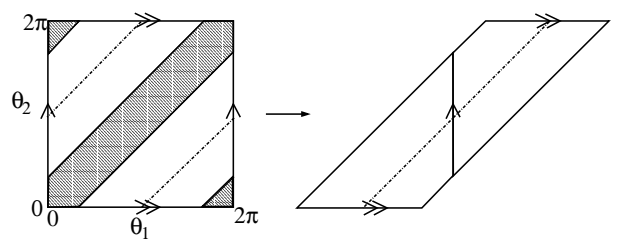

FIG. 3.6. $\mathbb{T}_{0}^{2}$ as a subset of $\mathbb{T}^{2}$ in the $\left(\theta_{1}, \theta_{2}\right)$ coordinate.

Each $\left(\theta_{1}, \theta_{2}\right) \in \mathbb{T}^{2}$ determines a unique point $f\left(\theta_{1}, \theta_{2}\right)$ in $Q$ by

$$
\begin{aligned}
f\left(\theta_{1}, \theta_{2}\right)=\frac{1}{3}\left(2 \cos \theta_{1}-\cos \theta_{2}, 2 \sin \theta_{1}-\sin \theta_{2},-\cos \theta_{1}+2 \cos \theta_{2},\right. \\
\left.-\sin \theta_{1}+2 \sin \theta_{2},-\cos \theta_{1}-\cos \theta_{2},-\sin \theta_{1}-\sin \theta_{2}\right)^{T},
\end{aligned}
$$

which is an embedding of $\mathbb{T}^{2}$ (respectively, $\mathbb{T}_{0}^{2}$ ) into $\mathbb{R}^{6}$ whose image is $Q$ (respectively, $\left.Q_{0}\right)$.

By using $f$ as the coordinate map, it can be verified that in the $\left(\theta_{1}, \theta_{2}\right)$ coordinate, equation (3.15) is reduced to the following second order ODE:

$$
\left\{\begin{array}{l}
2 \ddot{\theta}_{1}-\cos \left(\theta_{1}-\theta_{2}\right) \ddot{\theta}_{2}=\sin \left(\theta_{1}-\theta_{2}\right)\left(\dot{\theta}_{2}\right)^{2} \\
2 \ddot{\theta}_{2}-\cos \left(\theta_{1}-\theta_{2}\right) \ddot{\theta}_{1}=-\sin \left(\theta_{1}-\theta_{2}\right)\left(\dot{\theta}_{1}\right)^{2} .
\end{array}\right.
$$

Equation (3.17) is the geodesics equation of $\mathbb{T}^{2}$ with a suitably chosen metric $g$. In fact, let $\mathbb{R}^{6}$ be equipped with the standard Riemannian metric. $Q$ as a submanifold inherits from $\mathbb{R}^{6}$ a metric by restriction. Let $g$ be the corresponding metric on $\mathbb{T}^{2}$ obtained by pulling back the metric on $Q$ via $f$, so that $f$ becomes an isometry. Then, it can be proved (see Appendix B) that (3.17) is indeed the equation for geodesics of $\mathbb{T}^{2}$ under the metric $g$. As a result, each solution $\alpha^{*}$ of equation (3.15) is a geodesic of $Q$, which is not surprising by Remark 1 . Since $\mathbb{T}^{2}$ (hence $Q$ ) is compact, a solution to equation (3.15) is defined for all duration of time, provided that it stays inside $Q_{0}$. Equation (3.17) can be solved by two integrals, see [13] for details.

Deeper optimality conditions of conflict-free maneuvers can be obtained in this interpretation. For example, it is computed in Appendix $B$ that at each point $\left(\theta_{1}, \theta_{2}\right) \in \mathbb{T}^{2}$ the sectional curvature of the tangent plane spanned by the basis $\frac{\partial}{\partial \theta_{1}}$ and $\frac{\partial}{\partial \theta_{2}}$ at that point is

$$
K\left(\theta_{1}, \theta_{2}\right)=\frac{-9 \cos \left(\theta_{1}-\theta_{2}\right)}{\left[4-\cos ^{2}\left(\theta_{1}-\theta_{2}\right)\right]^{2}}
$$

Now consider the curve $\theta$ in $\mathbb{T}^{2}$ defined by $\theta(t)=\left(\theta_{1}(t), \theta_{2}(t)\right)=(t, \pi+t)$ for $t \in[0, \tau]$, where $\tau$ is positive. $\theta$ is a trivial solution to $(3.17)$, hence a geodesic of $\mathbb{T}^{2}$ that is contained completely in $\mathbb{T}_{0}^{2}$. $\theta$ determines a 3 -maneuver $\alpha^{*}=f \circ \theta$, i.e.

$$
\alpha_{1}^{*}(t)=(\cos t, \sin t)^{T}, \alpha_{2}^{*}(t)=(-\cos t,-\sin t)^{T}, \alpha_{3}^{*}(t)=(0,0)^{T}, t \in[0, \tau] .
$$

In the motions specified by $\alpha^{*}$, agent 3 stays at the origin, while agent 1 and agent 2 are at unit distance from agent 3 but on the opposite side of it so that three of them are always collinear, and both agent 1 and agent 2 rotate at the same constant angular velocity around agent 3. $\alpha^{*}$ thus defined is a solution to equation (3.15). 
An application of Proposition 3.8 implies that $\alpha^{*}$ is no longer optimal if $\tau>\pi$, for otherwise a better maneuver can be obtained by rotating agent 1 and agent 2 the opposite way around agent 3 . The following proposition improves this result.

Proposition 3.14. Maneuver $\alpha^{*}$ defined by (3.19) is not optimal if $\tau>\frac{\sqrt{2}}{2} \pi$.

Proof. Since $f$ is an isometry, we need only to prove that the geodesic $\theta$ is no longer distance-minimizing between its end points $\theta(0)=(0, \pi)$ and $\theta(\tau)=(\tau, \pi+\tau)$ once $\tau>\tau_{0}=\frac{\sqrt{2}}{2} \pi$. To this end, it suffices to prove that $\theta\left(\tau_{0}\right)$ is a conjugate point of $\theta(0)$ along $\theta$, in other words, there exists a nontrivial Jacobi field $X$ along $\theta$ that vanishes at both $\theta(0)$ and $\theta\left(\tau_{0}\right)([20])$.

Define two vector fields along $\theta$ by $W_{1}=\frac{\partial}{\partial \theta_{1}}+\frac{\partial}{\partial \theta_{2}}$ and $W_{2}=\frac{\partial}{\partial \theta_{1}}-\frac{\partial}{\partial \theta_{2}}$. Then, it is easy to verify that $W_{1}$ and $W_{2}$ are orthogonal, and that $W_{1}$ coincides with the velocity field $\dot{\theta}$ of the geodesic $\theta$. Moreover, using the Christoff symbols calculated in Appendix B, we conclude that $\nabla_{\dot{\theta}} W_{2} \equiv 0$; hence, $W_{2}$ is parallel along $\theta$.

A Jacobi field $X$ along $\theta$ and orthogonal to $\dot{\theta}$ is necessarily of the form $X(t)=$ $h(t) W_{2}(t)$ for some function $h$ defined on $[0, \tau]$, and satisfies the Jacobi equation $\nabla_{\dot{\theta}} \nabla_{\dot{\theta}} X+R(\dot{\theta}, X) \dot{\theta}=0$, where $R$ is the curvature tensor of $\mathbb{T}^{2}$. Since $\nabla_{\dot{\theta}} \nabla_{\dot{\theta}} X=$ $\ddot{h} W_{2}$ and $R(\dot{\theta}, X) \dot{\theta}$ are both orthogonal to $\dot{\theta}$, the Jacobi equation is equivalent to $\left\langle\ddot{h} W_{2}, W_{2}\right\rangle+\left\langle R\left(\dot{\theta}, h W_{2}\right) \dot{\theta}, W_{2}\right\rangle=0$. By (3.18), the sectional curvature $K$ of $\mathbb{T}^{2}$ along $\theta$ is constant 1 . Using the relation $\left\langle R\left(\dot{\theta}, h W_{2}\right) \dot{\theta}, W_{2}\right\rangle=h K\left[\langle\dot{\theta}, \dot{\theta}\rangle\left\langle W_{2}, W_{2}\right\rangle-\left\langle\dot{\theta}, W_{2}\right\rangle^{2}\right]$, we have $\ddot{h}+2 h=0$. A solution of $h$ vanishing at 0 is $h(t)=\sin (\sqrt{2} t)$, so $X(t)=$ $\sin (\sqrt{2} t) W_{2}(t)$ is an Jacobi field along $\theta$ vanishing at $t=0$ and $t=\frac{\sqrt{2}}{2} \pi=\tau_{0}$. Therefore, $\theta\left(\tau_{0}\right)$ is a conjugate point of $\theta(0)$ along $\theta$. $\mathbf{u}$

A more intuitive way of obtaining the conclusion of Proposition 3.14 is through variational analysis of $\alpha^{*}$ using perturbations of the following form. Recall that $\theta(t)=\left(\theta_{1}(t), \theta_{2}(t)\right)=(t, \pi+t), t \in T=[0, \tau]$ is the curve in $\mathbb{T}_{0}^{2}$ that $\alpha^{*}$ corresponds to. Let $\xi_{1}: T \times(-\epsilon, \epsilon) \rightarrow \mathbb{R}$ be a proper variation of the map $\theta_{1}: T \rightarrow \mathbb{R}$, i.e. $\xi_{1}$ is a smooth map such that $\xi_{1}(t, 0)=\theta_{1}(t), \xi_{1}(0, s)=\theta_{1}(0), \xi_{1}(\tau, s)=\theta_{1}(\tau)$ for $t \in T$ and $s \in(-\epsilon, \epsilon)$, where $\epsilon$ is a small positive number. Let $\xi_{2}: T \times(-\epsilon, \epsilon) \rightarrow \mathbb{R}$ be a proper variation of the map $\theta_{2}: T \rightarrow \mathbb{R}$. Consider joint maneuvers $\beta_{s}$ defined in $\left(\theta_{1}, \theta_{2}\right)$ coordinate by $\left(\xi_{1}(\cdot, s), \xi_{2}(\cdot, s)\right)$ for $s \in(-\epsilon, \epsilon)$, which all start from $\alpha^{*}(0)$ and end in $\alpha^{*}(\tau)$. In the braid representation, $\hat{\beta}_{s}$ is obtained from $\hat{\alpha}^{*}$ by rotating the strings $\hat{\alpha}_{1}^{*}$ and $\hat{\alpha}_{2}^{*}$ by certain angles with respect to the string $\hat{\alpha}_{3}^{*}$ and then re-align the three strings to the origin. $\beta_{s}$ is conflict-free if the variations $\xi_{1}$ and $\xi_{2}$ are small enough. Then, a necessary condition for $\alpha^{*}$ to be optimal is that the $\mu$-energy of $\beta_{s}$ is minimized at $s=0$ for all possible $\xi_{1}$ and $\xi_{2}$. After a lengthy calculation, this will lead to the conclusion of Proposition 3.14.

If we consider only conflict-free maneuvers with this particular contact graph, then it is proved in [17] that, after $\tau$ passes the critical value $\frac{\sqrt{2}}{2} \pi$, the optimal conflict-free maneuver from $\alpha^{*}(0)$ to $\alpha^{*}(\tau)$ bifurcates from $\alpha^{*}$ into two conflict-free maneuvers with identical energy. Shown in the first row of Fig. 3.7 are the plots of $\alpha^{*}$ for some $\tau>\frac{\sqrt{2}}{2} \pi$. The middle column is its plot in $\left(\theta_{1}, \theta_{2}\right)$ coordinate, and the right column is its braid representation. In the second and third rows, we plot by numerical simulations the two bifurcated optimal conflict-free maneuvers with this contact graph, which in $\left(\theta_{1}, \theta_{2}\right)$ coordinate are mirror image of each other with respect to the line $\theta_{1}-\theta_{2}=\frac{\pi}{2}$. For more details on the above claims and the general problem of conjugate points in manifolds with boundary, see [17].

One can also consider $n \geq 3$ agents with equal priorities, which are originally in a straight line with distance between successive agents being $R$, and which rotate at a 

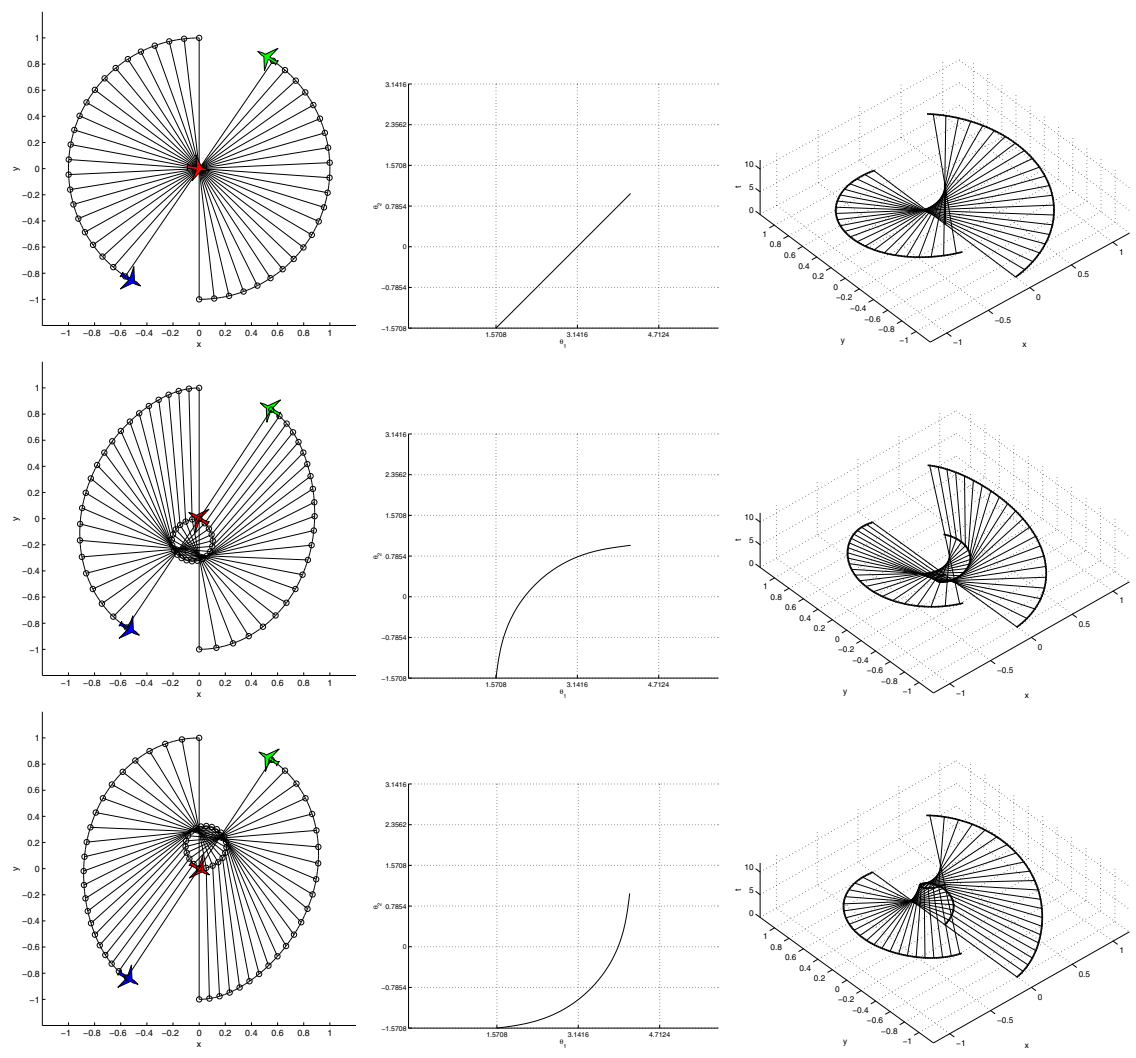

FIG. 3.7. Bifurcation of minimizing geodesics in $\mathbb{T}^{2}$. Left column: 3-maneuvers; Middle column: $\left(\theta_{1}, \theta_{2}\right)$ phase plots; Right column: braids.

constant angular velocity around their centroid. This defines a geodesic in a certain submanifold of $\mathbb{R}^{2 n}$ as we have discussed before. The maximal angle they can rotate before the first conjugate point of this geodesic is encountered in the submanifold is denoted by $\tau_{n}^{*}$. It can be expected that $\tau_{n}^{*}$ decreases with $n$. We conjecture that $\tau_{n}^{*}=\pi / \sqrt{\frac{n(n-1)}{2}-1}$. The case $n=3$ is proved in Proposition 3.14. The cases $n=4,5,6,7$ are verified symbolically using MAPLE.

It is worthwhile at this point to summarize the optimality conditions we have derived so far. All of them, with the exception of Proposition 3.8 and Proposition 3.14, are local in the sense that they can be obtained by using spike-like perturbations in the variational analysis, which only change maneuvers in a neighborhood of a fixed time epoch. Proposition 3.14 is semi-global in that its conclusion can only be reached by perturbations that change maneuvers throughout a subinterval of the encounter with positive length. Proposition 3.8 is the only global one, in the sense that it enables us to compare the performance of maneuvers belonging to different homotopy types.

3.7. Regularity of optimal conflict-free maneuvers. The regularity of optimal conflict-free maneuvers is a tricky issue. For example, it is unknown whether for each optimal $\alpha^{*}$, there exists a finite subdivision of $T, t_{0}<t_{1}<\cdots<t_{m}=t_{f}$, such that the contact graph $G_{\alpha^{*}}(t)$ remains constant during each subinterval $\left(t_{k}, t_{k+1}\right)$, and contiguous subintervals correspond to different contact graphs. It is proved in [2] 
that, in a Euclidean space under the presence of open obstacles with locally analytic boundary, a geodesic can have, in any segment of finite arc length, only a finite number of switch points where it switches from an interior segment to a segment on the boundary of an obstacle or vice versa. Unfortunately, this result does not apply in our case, since the obstacle $W$ as defined in Remark 1 has nonsmooth boundary.

On the other hand, it can be proved that an optimal $\alpha^{*}$ is always $C^{1}$, i.e. there is no sharp turns in the optimal conflict-free maneuvers. In fact, this follows from a general result proved in [13], which states that if a manifold $M$ with (nonsmooth) boundary is a subset of $\mathbb{R}^{k}$ obtained by removing from $\mathbb{R}^{k}$ a finite union of open convex subsets, each of which has a smooth boundary, then any geodesic of $M$ is of class $C^{1}$. Note that the convex subsets are not required to be disjoint for this conclusion. In our case, by Remark 1, the obstacle is the union of $\frac{n(n-1)}{2}$ convex cylinders in $\mathbb{R}^{2 n}$.

3.8. Two mechanical analogies. We now give two mechanical analogies of the above results. It should be pointed out that they serve only as analogies to gain more insights into the results obtained, and are not rigorous proofs themselves.

First, consider the following experiment. Instead of $n$ agents, we have $n$ particles of mass $\mu_{1}, \ldots, \mu_{n}$ on a horizontal plane with no external forces acting on them. At time $t_{0}$, they are at the initial positions $a_{1}, \ldots, a_{n}$ with certain initial velocities. Each particle $i$ moves with constant velocity until the distance between it and some other particle $j$ becomes $R$. Then a rigid rod of zero mass is introduced between particle $i$ and particle $j$ to prevent their distance from further decreasing, and the two particles move together with the rod at velocities determined by the law of conservation of momentum and angular momentum. We refer to the above process where a rigid rod is introduced between two particles as a (two-particle) join. There are two types of joins: tangential and non-tangential. A join is tangential if the time derivative of the distance between the two particles at the time of join is zero, otherwise the join is non-tangential. It is evident that some kinetic energy is lost for a non-tangential join since there is a collision between the two particles along the direction of the rod. As time goes on, more particles can join to form larger groups. In addition to joins, a group of particles connected by rods can split at any time, in the sense that some or all of the rods disappear instantly at that time. So when a split occurs, neither the positions nor the velocities of the particles change, but the group separates into several independent subgroups.

It is claimed that by appropriately choosing the initial velocities, time and order of the joins and splits, one can get from such an experiment the optimal maneuver $\alpha^{*}$. In fact, during any time interval $I$ in which there are neither joins nor splits, the system of particles naturally corresponds to a contact graph with edges between vertices representing rods between particles. Moreover, if $I$ is sufficiently small, the motions of the particles correspond to the optimal conflict-free maneuver associated with such a contact graph. To see this, recall that by the principle of least action ([3]), the motion of the interconnected particles system is an extremal of the action integral $\int_{I}(E-U) d t$. Here $E=\frac{1}{2} \sum_{i=1}^{n} \mu_{i} v_{i}^{2}$ represents the kinetic energy, and $U$ is the potential, which is zero by our assumption on the absence of external forces. So, for sufficiently small time interval $I$, the motions of the interconnected particles minimize $\frac{1}{2} \sum_{i=1}^{n} \mu_{i} \int_{I} v_{i}^{2} d t$; hence they specify precisely the optimal maneuver over $I$ by definition. Equation (3.15) determines, for example, the motions of three particles connected by two rigid rods with zero masses. For discussions on the general problem of kinematically coupled structures composed of rigid and flexible bodies, see [22] and other references in the same book. 


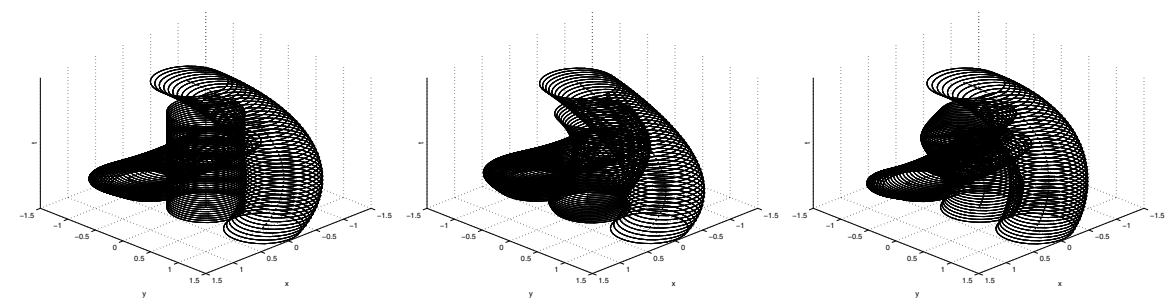

FIG. 3.8. Examples of elastic (enlarged) braids in equilibrium positions. Left: unstable; Center and right: stable.

In this mechanical interpretation, the conclusion of Proposition 3.2 is simply the invariance of the motions of a mechanical system with respect to changes of inertial coordinates. Since the total momentum and the total angular momentum of the system are conserved in each time interval with constant configuration (contact graph) and do not change during joins or splits, they are constant during the whole time interval $T$, which are the conclusions of Corollary 3.5 and Proposition 3.8, respectively. Proposition 3.8 further imposes an upper bound on the total angular momentum, implying that the whole system cannot spin "too fast". In addition, the assertion in Section 3.7 that $\alpha^{*}$ is $C^{1}$ implies that all the joins should be tangential; hence there is no kinetic energy lost during joints and splits and the total kinetic energy $\frac{1}{2} \sum_{i=1}^{n} \mu_{i} v_{i}^{2}$ is also conserved, as it is shown in [12] by using a reparameterization operator.

In mechanics, there is a systematic way of using symmetry on the configuration space to reduce the degree of freedom $([3,26])$. In our case, the symmetry is $\mathbf{S E}_{2}$, the group of rigid motions in $\mathbb{R}^{2}$, acting on $\mathbb{R}^{2}$. Hence the analysis leading to Corollary 3.5 and Proposition 3.8 (except the bound in Proposition 3.8) is simply the application of the symmetry reduction method uniformly to all the configuration spaces of a system with time-varying configurations. Compared with more advanced techniques such as those based on the Hamiltonian, symplectic, and Poisson viewpoints, our approach, which is Lagrangian in nature, deals with the nonsmoothness of the boundary constraints directly, thus avoiding the trouble of solving for each smooth component of the boundary constraints individually before piecing them together properly to get the final solution. In [16], the corresponding method is generalized to an arbitrary Riemannian manifold with a group of isometries. For application of Lagrangian reduction to holonomic and nonholonomic mechanical systems, see [21].

A major drawback of the above mechanical model is that it is local, hence little insights can be obtained about the global optimality conditions. In this sense, the second model we are going to present is more "faithful", and once again demonstrates the advantage of adopting the braid point of view. As we have shown in Section 2, each conflict-free maneuver $\alpha$ of the $n$ agents corresponds to an $n$-braid $\hat{\alpha}$, whose intersection with any horizontal plane $t=\tau(\tau \in T)$ consists of $n$ points satisfying the $R$-separation property. Therefore, if we enlarge the radius of strings in $\hat{\alpha}$ to $R / 2$, or more precisely, if we think of each of the $n$ strings in $\hat{\alpha}$ as consisting of an infinite number of horizontal disks of radius $R / 2$ and height 0 mounting vertically, with each disk confined to move in a fixed horizontal plane $t=t_{1}$ for some $t_{1} \in T$, then the condition that $\alpha$ is conflict-free is equivalent to that the $n$ enlarged strings in $\hat{\alpha}$ do not overlap. Examples of such enlarged braids are shown in Fig. 3.8 for the three conflict-free maneuvers in Fig. 3.7.

Assume that, for each $i=1, \ldots, n$, the enlarged string $\hat{\alpha}_{i}$ in $\hat{\alpha}$ is elastic with 
elasticity coefficient $\mu_{i}$, and has smooth surface so that any two strings can slide along each other without friction. Under these assumptions, the elastic energy of this $n$-string system is proportional to the $\mu$-energy of the corresponding conflictfree maneuver. If we fix the strings in $\hat{\alpha}$ at both the bottom $\left(t=t_{0}\right)$ and the top $\left(t=t_{f}\right)$ horizontal planes and leave free the remaining parts, then for certain choices of $\alpha$ this elastic $n$-string system will be in an equilibrium (stationary) position. The optimal conflict-free maneuvers have minimal energy, hence necessarily correspond to equilibrium positions.

Suppose that $\hat{\alpha}$ is in an equilibrium position. Pick any disk in $\hat{\alpha}$ that belongs to the string $\hat{\alpha}_{i}$ and lies on the horizontal plane $t=t_{1}$ for some $i=1, \ldots, n$ and $t_{0}<t_{1}<t_{f}$. Denote this disk by $D_{i}\left(t_{1}\right)$. Then $D_{i}\left(t_{1}\right)$ is subject to two types of forces: forces enacted by disks in the same string that are immediately above and below $D_{i}\left(t_{1}\right)$, i.e. $D_{i}\left(t_{1}^{+}\right)$and $D_{i}\left(t_{1}^{-}\right)$; and forces enacted by disks in the same horizontal plane $t=t_{1}$ but belonging to different strings, i.e. $D_{j}\left(t_{1}\right)$ with $j \neq i$. Since $D_{i}\left(t_{1}\right)$ is confined to move on the plane $t=t_{1}$, we are concerned with only the projection of the forces onto this plane. The contribution of the forces of the first type is easily seen to be proportional to $\mu_{i} \ddot{\alpha}_{i}\left(t_{1}\right)$. As for the forces of the second type, say, the force enacted by disk $D_{j}\left(t_{1}\right)(j \neq i)$ that contacts $D_{i}\left(t_{1}\right)$, by our assumption of no frictions, this force is directed from the center of $D_{j}\left(t_{1}\right)$ to the center of $D_{i}\left(t_{1}\right)$, i.e. from $\left(\alpha_{j}\left(t_{1}\right), t_{1}\right)$ to $\left(\alpha_{i}\left(t_{1}\right), t_{1}\right)$. Now the conclusion of Proposition 3.13 can be explained as follows. Let $\mathcal{I}$ be a subset of $\{1, \ldots, n\} \backslash\{i\}$ that corresponds to a maximal connected component of the graph obtained by removing node $i$ and all the edges connected with it from the contact graph of $\alpha$ at time $t_{1}$. Since $\hat{\alpha}$ is in an equilibrium position, the subsystem $D_{\mathcal{I}}\left(t_{1}\right)$ consisting of disks $D_{j}\left(t_{1}\right)$ for $j \in \mathcal{I}$ is stationary. So the total moment (torque) of external forces acting on $D_{\mathcal{I}}\left(t_{1}\right)$ is zero, which is exactly the conclusion of Proposition 3.13. Note that here we choose $\left(\alpha_{i}\left(t_{1}\right), t_{1}\right)$ as the origin and use the fact that torque of forces enacted by $D_{i}\left(t_{1}\right)$ on disks in $D_{\mathcal{I}}\left(t_{1}\right)$ is zero by our above analysis.

Other optimality conditions can also be explained in this model. For example, the conclusion of Corollary 3.5 is, after differentiation with respect to $t$ twice, simply that on any horizontal plane $t=t_{1}, t_{0}<t_{1}<t_{f}$, the combined external forces acting on the subsystem consisting of disks $D_{i}\left(t_{1}\right), i=1, \ldots, n$, is zero. For the example in Section 3.6, the semi-global conclusion of Proposition 3.14 can be intuitively understood as that, after a rotation of more than $\frac{\pi}{\sqrt{2}}$, the cumulative force of the two neighboring strings on the central one exceeds the critical value so that the equilibrium position of $\hat{\alpha}^{*}$ becomes unstable. Any slight perturbation will then render the system to settle in one of the two bifurcated positions with minimal elastic energy (see Fig. 3.8), provided that there exists very small but nonzero air frictions to avoid persistent oscillation.

4. Optimal multi-legged conflict-free maneuvers. Due to the difficulty in computing analytically the optimal conflict-free maneuver when the number $n$ of agents is greater than two, we now restrict our attention to those maneuvers specified by a set of waypoints, which might well be the only feasible form of joint maneuvers that a central controller can specify to the participating agents in practice.

To be precise, consider $n$ agents with starting position $\mathbf{a}=\left(a_{1}, \cdots, a_{n}\right)$ and destination position $\mathbf{b}=\left(b_{1}, \cdots, b_{n}\right)$. Assume that a set of epochs $\left\{t_{j}\right\}_{j=0}^{m}, t_{0}<t_{1}<$ $\cdots<t_{m-1}<t_{m}=t_{f}$, where $m$ is a positive integer, has been fixed. For each agent $i$, choose a set of waypoints $\left\{c_{i, j}\right\}_{j=0}^{m}$ in $\mathbb{R}^{2}$ such that $c_{i, 0}=a_{i}$ and $c_{i, m}=b_{i}$. Then, an $m$-legged maneuver of agent $i$ is a maneuver consisting of $m$ stages, where at each 
stage $j \in\{0,1, \cdots, m-1\}$, agent $i$ starts from $c_{i, j}$ at time $t_{j}$ and reaches $c_{i, j+1}$ at time $t_{j+1}$ with constant velocity. Denote by $\mathbf{P}_{i}^{m}$ the set of all $m$-legged maneuvers of agent $i$, and by $\mathbf{P}^{m}(\mathbf{a}, \mathbf{b})=\prod_{i=1}^{n} \mathbf{P}_{i}^{m}$ the set of all $m$-legged joint maneuvers. In the braid representation, an $m$-legged joint maneuver corresponds to $n$ strings, each one consisting of $m$ line segments pieced together. The set of $m$-legged conflict-free maneuvers consists of all elements of $\mathbf{P}^{m}(\mathbf{a}, \mathbf{b})$ with MSE at least $R$ and is denoted by $\mathbf{P}^{m}(R, \mathbf{a}, \mathbf{b})$.

In this section, we shall try to solve the following version of problem (3.4):

$$
\text { minimize } J_{\mu}(\alpha) \text { subject to } \alpha \in \mathbf{P}^{m}(R, \mathbf{a}, \mathbf{b}) \text {. }
$$

By using similar arguments, one can show that some of the optimality conditions in Section 3, such as Corollary 3.5, still apply for solutions to problem (4.1). In general, a solution to problem (4.1) is only suboptimal for problem (3.4).

4.1. Optimal 2-legged conflict-free maneuver for two agents. We start from the simplest case when $n=2$ and $m=2$. Consider two agents with starting position $\mathbf{a}=\left(a_{1}, a_{2}\right)$ and destination position $\mathbf{b}=\left(b_{1}, b_{2}\right)$. Let $\alpha=\left(\alpha_{1}, \alpha_{2}\right)$ be a 2-legged conflict-free maneuver in $\mathbf{P}^{2}(R, \mathbf{a}, \mathbf{b})$ with three waypoints $c_{i, j}, j=0,1,2$, for each agent $i=1,2$. Since $c_{i, 0}=a_{i}$ and $c_{i, 2}=b_{i}$ are fixed for each agent $i$, the middle waypoints $c_{i, 1}$ will be denoted by $c_{i}$ to simplify the notations. Let $t_{c} \in\left(t_{0}, t_{f}\right)$ be the epoch corresponding to the middle waypoints. Then, the motions of the two agents are described by

$$
\alpha_{i}(t)=\left\{\begin{array}{ll}
a_{i}+\left(c_{i}-a_{i}\right) \frac{t-t_{0}}{t_{c}-t_{0}}, & t_{0} \leq t \leq t_{c} \\
b_{i}+\left(c_{i}-b_{i}\right) \frac{t-t_{f}}{t_{c}-t_{f}}, & t_{c} \leq t \leq t_{f}
\end{array}, \quad i=1,2\right.
$$

After some calculations, the $\mu$-energy of a maneuver $\alpha \in \mathbf{P}^{2}(\mathbf{a}, \mathbf{b})$ as the function of $c_{1}$ and $c_{2}$ can be expressed as follows

$$
J_{\mu}(\alpha)=\frac{t_{f}-t_{0}}{\left(t_{f}-t_{c}\right)\left(t_{c}-t_{0}\right)}\left[\mu_{1}\left\|c_{1}-c_{1}^{u}\right\|^{2}+\mu_{2}\left\|c_{2}-c_{2}^{u}\right\|^{2}\right]+C,
$$

where $C$ is a constant and $c_{i}^{u}, i=1,2$, are defined by

$$
c_{i}^{u}=\frac{\left(t_{f}-t_{c}\right) a_{i}+\left(t_{c}-t_{0}\right) b_{i}}{t_{f}-t_{0}}, \quad i=1,2 .
$$

Note that $c_{1}^{u}$ and $c_{2}^{u}$ are the optimal waypoints when minimizing $J_{\mu}(\alpha)$ without the MSE constraint. In the braid representation, $c_{1}^{u}$ and $c_{2}^{u}$ correspond to the intersections of the plane $t=t_{c}$ with the lines joining $\left(a_{i}, t_{0}\right)$ to $\left(b_{i}, t_{f}\right)$, for $i=1$ and 2 , respectively.

The MSE constraint can be simplified as well. The minimal distance $d_{l}$ between the two agents during the time interval $\left[t_{0}, t_{c}\right]$ is given by

$$
d_{l}= \begin{cases}\left\|c_{1}-c_{2}\right\|, & \text { if } \lambda<-\left\|c_{1}-c_{2}-a_{1}+a_{2}\right\|^{2} \\ \sqrt{\left\|a_{1}-a_{2}\right\|^{2}-\lambda^{2} /\left\|c_{1}-c_{2}-a_{1}+a_{2}\right\|^{2}}, & \text { if }-\left\|c_{1}-c_{2}-a_{1}+a_{2}\right\|^{2} \leq \lambda \leq 0 \\ \left\|a_{1}-a_{2}\right\|, & \text { if } \lambda>0,\end{cases}
$$

where $\lambda \triangleq\left(a_{1}-a_{2}\right)^{T}\left(c_{1}-c_{2}-a_{1}+a_{2}\right)$. Note that $d_{l}$ is a function of the relative positions $a_{1}-a_{2}$ and $c_{1}-c_{2}$ only and is independent of the epoch $t_{c}$. We then use $d_{l}\left(a_{1}-a_{2}, c_{1}-c_{2}\right)$ to denote it explicitly. Similarly, the minimum distance between the two agents during the time interval $\left[t_{c}, t_{f}\right]$ is $d_{l}\left(c_{1}-c_{2}, b_{1}-b_{2}\right)$. 


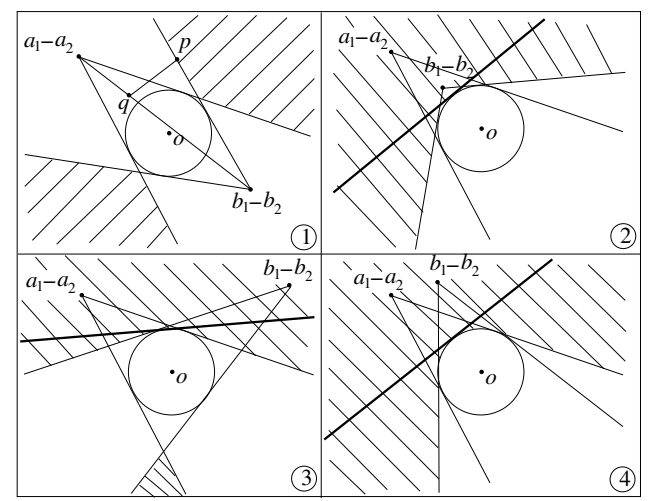

FIG. 4.1. The four configurations of the feasible set $A$ for $c_{1}-c_{2}$.

For $\alpha$ to be a conflict-free maneuver, both $d_{l}\left(a_{1}-a_{2}, c_{1}-c_{2}\right)$ and $d_{l}\left(c_{1}-c_{2}, b_{1}-\right.$ $b_{2}$ ) have to be at least $R$, yielding two constraints on $c_{1}-c_{2}$. Depending on the relative position of $a_{1}-a_{2}$ and $b_{1}-b_{2}$, the feasible set $A$ for $c_{1}-c_{2}$ has four possible configurations, which are numbered from 1 to 4 and represented by shaded regions in Fig. 4.1. Notice that $A$ consists of two connected components in configuration 1 and 3 , which correspond to the two fundamental types of the conflict-free maneuvers. In configurations 2 and 4 , however, only one fundamental type can be achieved by 2-legged maneuvers.

REMARK 5. The feasible set $A$ for $c_{1}-c_{2}$ can be characterized as the subset of $\mathbb{R}^{2}$ consisting of all those points that are "visible" to both $a_{1}-a_{2}$ and $b_{1}-b_{2}$ in the presence of the open disk $B(0, R)$ as obstacle. In fact, by applying an appropriate tilt operator $\mathcal{T}_{w}$ that preserves the MSE and $c_{1}-c_{2}$, one can assume that $c_{2}=a_{2}$, i.e., agent 2 stays at $a_{2}$ during $\left[t_{0}, t_{c}\right]$. Thus the MSE constraint during $\left[t_{0}, t_{c}\right]$ is equivalent to the constraint that the line segment from $a_{1}$ to $c_{1}$ does not intersect $B\left(a_{2}, R\right)$, or alternatively, the line segment from $a_{1}-a_{2}$ to $c_{1}-c_{2}$ does not intersect $B(0, R)$. Similar arguments apply to the second stage of $\alpha$.

As a result of the above simplifications, problem (4.1) is reduced to

$$
\text { minimize } \mu_{1}\left\|c_{1}-c_{1}^{u}\right\|^{2}+\mu_{2}\left\|c_{2}-c_{2}^{u}\right\|^{2} \text { subject to } c_{1}-c_{2} \in A \text {. }
$$

Theorem 4.1. Define $q \triangleq c_{1}^{u}-c_{2}^{u}=\frac{t_{f}-t_{c}}{t_{f}-t_{0}}\left(a_{1}-a_{2}\right)+\frac{t_{c}-t_{0}}{t_{f}-t_{0}}\left(b_{1}-b_{2}\right)$. Let $p$ be $a$ point in A at minimum distance from q. An optimal solution to problem (4.4) is then given by

$$
c_{1}^{*}=\mu_{1} c_{1}^{u}+\mu_{2} c_{2}^{u}+\mu_{2} p, \quad c_{2}^{*}=\mu_{1} c_{1}^{u}+\mu_{2} c_{2}^{u}-\mu_{1} p .
$$

Moreover, if problem (4.4) is restricted to one of the two fundamental types of conflictfree maneuvers that is achievable by 2-legged maneuvers, then $c_{1}^{*}$ and $c_{2}^{*}$ are unique.

Proof. Set $\Delta c=c_{1}-c_{2}$. Then we have

$$
\begin{aligned}
& \min \left\{\mu_{1}\left\|c_{1}-c_{1}^{u}\right\|^{2}+\mu_{2}\left\|c_{2}-c_{2}^{u}\right\|^{2}: c_{1}, c_{2} \text { such that } \Delta c \in A\right\} \\
& =\min _{\Delta c \in A} \min _{c_{2}}\left\{\mu_{1}\left\|c_{2}+\Delta c-c_{1}^{u}\right\|^{2}+\mu_{2}\left\|c_{2}-c_{2}^{u}\right\|^{2}\right\} \\
& =\min _{\Delta c \in A} \min _{c_{2}}\left\{\left\|c_{2}-\mu_{1}\left(c_{1}^{u}-\Delta c\right)-\mu_{2} c_{2}^{u}\right\|^{2}+\mu_{1} \mu_{2}\left\|c_{1}^{u}-c_{2}^{u}-\Delta c\right\|^{2}\right\} \\
& =\min _{\Delta c \in A} \mu_{1} \mu_{2}\|q-\Delta c\|^{2} \\
& =\mu_{1} \mu_{2}\|q-p\|^{2}
\end{aligned}
$$



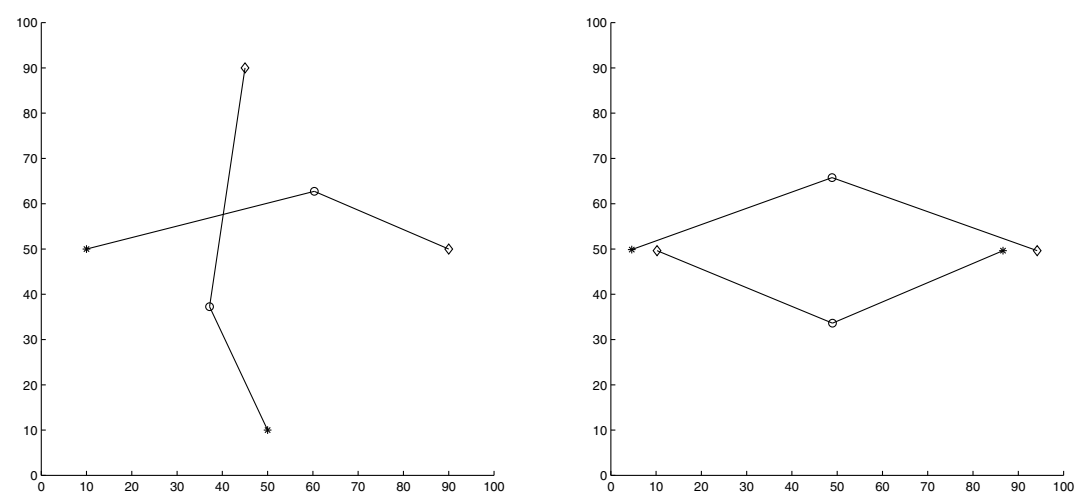

FIG. 4.2. 2-legged optimal conflict-free maneuvers for 2-agent encounters $\left(\mu_{1}=\mu_{2}=0.5, R=30\right)$.

where the last two equalities follow by choosing $c_{2}=\mu_{1}\left(c_{1}^{u}-\Delta c\right)+\mu_{2} c_{2}^{u}$ and $\Delta c=p$. Together they imply the desired expressions of $c_{1}^{*}$ and $c_{2}^{*}$. The uniqueness of $c_{1}^{*}$ and $c_{2}^{*}$ given a particular fundamental type is a consequence of the fact that $p$ is unique, since either the connected component of $A$ corresponding to that type is convex, or $q$ is contained in it since it lies on the line segment connecting $a_{1}-a_{2}$ to $b_{1}-b_{2}$.

Note that in configuration 2,3 , and $4, p=q$ since $q$ lies on the line segment connecting $a_{1}-a_{2}$ and $b_{1}-b_{2}$ that is contained entirely in $A$. Hence $c_{1}^{*}$ and $c_{2}^{*}$ are equal to $c_{1}^{u}$ and $c_{2}^{u}$, respectively. In configuration 1 , the set $A$ is the union of two disjoint convex sets, so there might be up to two points in $A$ nearest to $q$, with two being the case when there is an exact collision for the unconstrained optimal joint maneuver. In this case, we can choose either of the two points as $p$.

Fig. 4.2 shows the optimal 2-legged conflict-free maneuvers for some typical 2agent encounters when the agents have equal priorities. In each plot, the starting points are marked with stars and the ending points with diamonds. The circles are the waypoints specified by Theorem 4.1.

4.2. Optimal 2-legged conflict-free maneuver for multiple agents. Consider the case $m=2$ and $n \geq 3$. Roughly speaking, the nature of problem (4.1) is mainly combinatorial in that the major task is to choose the type of conflict-free maneuvers in which one can find the optimal solution. In this section, we deal only with the problem of finding the optimal conflict-free maneuver within a given type. We postpone to Section 4.4 the discussion on how to choose the maneuver type.

Fix $t_{c} \in\left(t_{0}, t_{f}\right)$ and denote by $A_{i j}$ the feasible set for $c_{i}-c_{j}$ when only the agent pair $(i, j)$ is present. $A_{i j}$ is computed as set $A$ in the last subsection with $a_{i}, b_{i}, a_{j}, b_{j}$ in the place of $a_{1}, b_{1}, a_{2}, b_{2}$. Suppose that we have chosen a type of conflict-free maneuvers. Then, the problem is to find the waypoints $c_{1}, \cdots, c_{n}$ that

$$
\text { minimize } \sum_{i=1}^{n} \mu_{i}\left\|c_{i}-c_{i}^{u}\right\|^{2} \text { subject to } c_{i}-c_{j} \in A_{i j}^{ \pm}, \quad 1 \leq i<j \leq n,
$$

where $c_{i}^{u}$ is defined as in (4.3) for $i=1, \cdots, n$, and $A_{i j}^{ \pm}$denotes the connected component of the set $A_{i j}$ matching the desired type. Note that only a finite subset of types of conflict-free maneuvers can be represented in this way, and we assume that the given type belongs to this subset.

Notice that in all but the first configuration shown in Fig. 4.1 representing $A_{i j}$ 

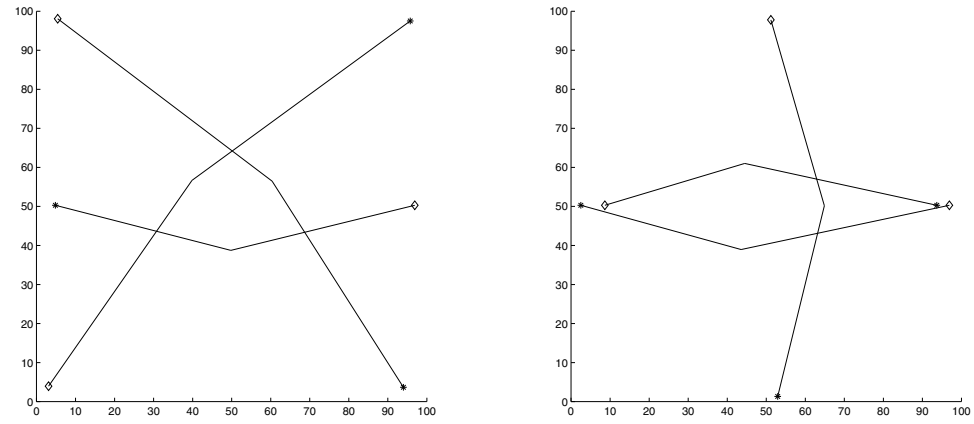

FIG. 4.3. Globally optimal 2-legged conflict-free 3-maneuvers $\left(\mu_{1}=\mu_{2}=0.5, R=20\right)$.

for $i=1$ and $j=2$, one of the connected components of $A_{i j}$ is nonconvex, posing great challenge for the efficient solution of problem (4.5). Therefore, in configuration 2,3 , and 4 , we linearize the nonconvex component of $A_{i j}$ by using a half space inner approximation, as it is shown in Fig. 4.1 by the black lines tangential to the boundary of $B(0, R)$. The choice of the black line may not be unique, and one should ensure that the inner approximated feasible region of $c_{i}-c_{j}$ contains the unconstrained optimal value $c_{i}^{u}-c_{j}^{u}$.

REMARK 6. Problem 4.5 is a linearly constrained convex optimization problem in the special case when any pair of agents is in the first configuration, i.e., when the unconstrained optimal joint maneuver will cause a conflict between any pair of agents. Therefore, our linear approximation scheme is tight for the most critical encounters.

After the linearization, if necessary, we have a linearly constrained quadratic optimization problem that can be solved efficiently. In the case when the number of agents is relatively small, we can afford the luxury of running the optimization algorithm for each type achievable by 2-legged maneuvers so as to find the globally optimal 2-legged conflict-free maneuver. Simulation results using MATLAB are shown in Fig. 4.3 for two 3-agent encounters. In both cases, each pair of agents is in the first configuration, so linearizations are not necessary and the obtained maneuvers are actually the globally optimal 2-legged conflict-free maneuvers.

4.3. Optimal $m$-legged conflict-free maneuver for multiple agents. The algorithm described in Section 4.2 can be used in an iterative way in the general case when the number $m$ of legs is greater than two. Fix a set of epochs $t_{0}<t_{1}<\ldots<$ $t_{m-1}<t_{m}=t_{f}$. A necessary condition for a set of waypoints $c_{i, j}, i=1, \ldots, n$, $j=0, \ldots, m$, with $c_{i, 0}=a_{i}, c_{i, m}=b_{i}$ to be an optimal solution to problem (4.1) is that

$$
c_{i, j}=c_{i}^{*}\left(\left(c_{1, j-1}, \ldots, c_{n, j-1}\right),\left(c_{1, j+1}, \ldots, c_{n, j+1}\right), t_{j-1}, t_{j}, t_{j+1}\right)
$$

for $1 \leq j \leq m-1$. Here $c_{i}^{*}\left(\left(c_{1, j-1}, \ldots, c_{n, j-1}\right),\left(c_{1, j+1}, \ldots, c_{n, j+1}\right), t_{j-1}, t_{j}, t_{j+1}\right)$ denotes the waypoint of agent $i$ for the optimal 2-legged maneuver when the starting and destination positions of the agents are $\left(c_{1, j-1}, \ldots, c_{n, j-1}\right)$ and $\left(c_{1, j+1}, \ldots, c_{n, j+1}\right)$, and the starting, middle and ending epochs are $t_{j-1}, t_{j}, t_{j+1}$, respectively. This condition inspires the following algorithm.

Algorithm 1.

1. Pick any feasible set of waypoints $c_{i, j}^{(0)}, 1 \leq i \leq n, 0 \leq j \leq m$, such that 

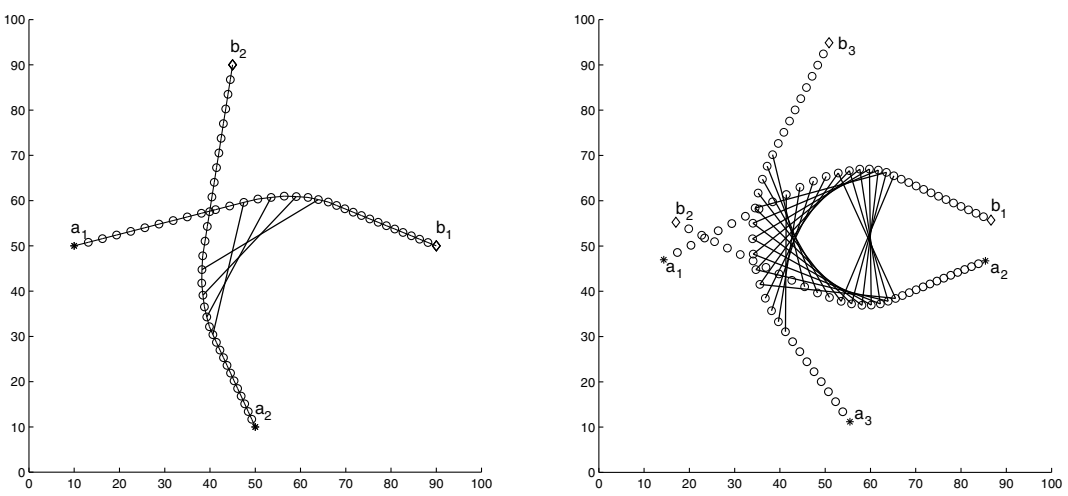

FIG. 4.4. Simulation results of Algorithm 1 for two and three agents encounters $(R=30)$.

$c_{i, 0}^{(0)}=a_{i}, c_{i, m}^{(0)}=b_{i}$ for $1 \leq i \leq n$ and such that the MSE constraint is satisfied over $T$.

2. For $j=1, \ldots, m-1$ compute for $i=1, \ldots, n$

$$
c_{i, j}^{(k+1)}=c_{i}^{*}\left(\left(c_{1, j-1}^{(k)}, \ldots, c_{n, j-1}^{(k)}\right),\left(c_{1, j+1}^{(k)}, \ldots, c_{n, j+1}^{(k)}\right), t_{j-1}, t_{j}, t_{j+1}\right) .
$$

3. Repeat procedure 2 with $k:=k+1$ until the decrease in $\mu$-energy is below some threshold $\varepsilon$.

It is easily seen that the $\mu$-energy of the conflict-free maneuvers obtained by Algorithm 1 is nonincreasing as a function of the iteration number $k$, and is strictly decreasing whenever condition (4.6) is not satisfied. Therefore, the iteration procedure converges asymptotically to a conflict-free maneuver satisfying condition (4.6). A convergence analysis of Algorithm 1 is yet to be achieved. Besides the issue of local minima suggested by the example in Section 3.6, the situation is further complicated by the fact that the convex optimization procedure introduced in Section 4.2 only yields an approximation of $c_{i}^{*}$. Another open issue is the suboptimality of optimal $m$-legged maneuvers in $\mathbf{P}^{m}(R, \mathbf{a}, \mathbf{b})$ with respect to optimal solutions in $\mathbf{P}(R, \mathbf{a}, \mathbf{b})$. Although in theory the performance gap decreases to zero as $m \rightarrow \infty$, in practice, it is not easy to quantify the performance degradation for a finite $m$.

In Fig. 4.4, some simulation results for Algorithm 1 when the agents have identical priorities and $R=30$ are shown. The epochs are chosen to evenly divide $\left[t_{0}, t_{f}\right]$, and the corresponding waypoints are marked with small circles. In the plots, whenever two agents are at distance $R$, their positions are joined by a line segment. Note that the result shown in the left figure is a good approximation to the optimal maneuvers plotted in Fig. 3.3.

4.4. Randomized optimization. In [12, 34], a decentralized algorithm for multi-agent conflict resolution is proposed in the context of air traffic control. By modeling the agent motion as a Brownian motion with drift, the probability of conflict between two agents is estimated and then used to generate repulsive forces between the agents, inspired by the potential and vortex field methodology for path planning $([27,36])$. Compared with traditional potential field methods that use only the positions of the agents, this algorithm considers also their headings and speeds, and hence generates maneuvers with less abrupt turns.

Although the stochastic algorithm can be run in real time regardless of the number $n$ of agents involved, one of its drawbacks is that absolute safety cannot be guaranteed 

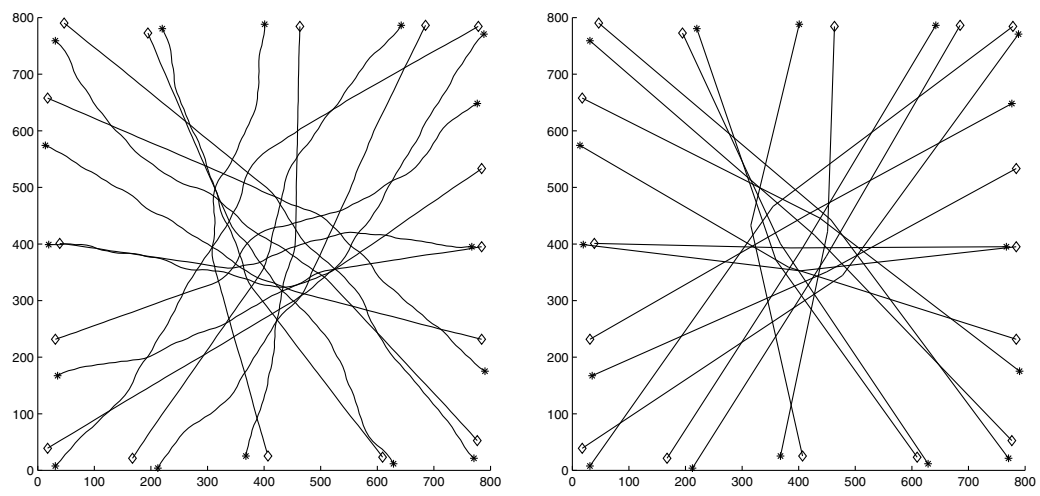

FIG. 4.5. 16-maneuvers generated by stochastic (left) and convex optimization algorithm (right).

with probability one. On the other hand, the convex optimization algorithm we propose in this paper can ensure absolute safety, but it cannot handle the explosively increasing number of types when $n$ is large. We then suggest a solution that combines the positive features of these two algorithms, hence it both guarantees safety and is computationally feasible. The proposed algorithm uses the stochastic algorithm as the random "type chooser". More specifically, for a given multi-agent encounter, first the stochastic algorithm is run to generate a joint maneuver corresponding to a particular type, and then the convex optimization algorithm is utilized to obtain an approximation of the optimal multi-legged maneuver within the type selected by the stochastic algorithm.

Simulation results for a 16-agent symmetric encounter are shown in Fig. 4.5, in which 16 agents with identical priorities pass approximatively through a common point at angles evenly distributed in $[0,2 \pi]$ and $R=30$. The one on the left is the joint maneuver generated by the stochastic algorithm, whereas the one on the right is the optimal 2-legged conflict-free maneuver within this type generated by the convex optimization algorithm.

REMARK 7. When the number of agents is small, say, $n=2,3$, experiments show that the stochastic algorithm tends to choose with higher probability those types with lower $\mu$-energy. However, when $n$ is large such as in the previous example, it is hard to evaluate the performance of the randomized algorithm, since currently no theoretical result exists that can exhaust the explosively increasing number of resolution types and find the optimal one (or ones). Much more work is needed in this respect.

5. Conclusions and future directions. In this paper, the problem of optimal coordinated motion planning for multiple agents moving on a plane is studied. After a classification of the homotopy types of conflict-free maneuvers, a weighted energy is proposed as the cost function to select the optimal one. Various local and global optimality conditions are derived. For two-agent encounters, analytical solutions are obtained both for the optimal continuous and piecewise- $C^{2}$ maneuvers and the optimal 2-legged maneuvers. For the general multi-agent case, a randomized convex optimization algorithm is proposed to find the optimal multi-legged maneuvers numerically.

To completely characterize the optimal conflict-free maneuvers, many issues remain to be solved. The results in this paper could serve as a good starting point. Possible directions of future research include the analysis of the proposed numerical 
algorithm in terms of its performance and its robustness with respect to uncertainty on the agents' positions and velocities, and the study of more realistic (and more complicated) models for the agent dynamics than the kinematic one adopted in this paper. Some contribution in this direction can be found in [15], which focuses exclusively on air traffic management systems.

Acknowledgment: The first author would like to thank Alan Weinstein for his insightful comments and discussions on the results of this paper.

Appendix A. Proof of Proposition 3.8.

Consider first the case when $s=0$. For each $\alpha \in \mathbf{P}(R, \mathbf{a}, \mathbf{b})$, let $\beta=\mathcal{R}_{\theta}^{0}(\alpha)$. Then

$$
\dot{\beta}_{i}(t)=T_{\theta(t)} \dot{\alpha}_{i}(t)+\frac{d}{d t} T_{\theta(t)} \alpha_{i}(t)=T_{\theta(t)} \dot{\alpha}_{i}(t)+\dot{\theta}(t) T_{\frac{\pi}{2}+\theta(t)} \alpha_{i}(t), \quad i=1, \cdots, n .
$$

Since $T_{\theta(t)}$ and $T_{\frac{\pi}{2}+\theta(t)}$ are orthonormal matrices and $T_{\frac{\pi}{2}+\theta(t)}^{T}=T_{-\frac{\pi}{2}-\theta(t)}$, we have

$$
\left\|\dot{\beta}_{i}(t)\right\|^{2}=\left\|\dot{\alpha}_{i}(t)\right\|^{2}+\left\|\alpha_{i}(t)\right\|^{2}|\dot{\theta}(t)|^{2}+2 \dot{\theta}(t) \alpha_{i}^{T}(t) T_{-\frac{\pi}{2}} \dot{\alpha}_{i}(t), \quad i=1, \cdots, n .
$$

Integrating and summing over $i$, we can write the cost difference $\Delta J_{\mu}(\theta)$ as

$$
\Delta J_{\mu}(\theta)=J_{\mu}(\beta)-J_{\mu}(\alpha)=\int_{t_{0}}^{t_{f}}\left[f(t)|\dot{\theta}(t)|^{2}+2 g(t) \dot{\theta}(t)\right] d t,
$$

where $f$ and $g$ are functions defined by

$$
f(t) \triangleq \frac{1}{2} \sum_{i=1}^{n} \mu_{i}\left\|\alpha_{i}(t)\right\|^{2}, \quad g(t) \triangleq \frac{1}{2} \sum_{i=1}^{n} \mu_{i} \alpha_{i}^{T}(t) T_{-\frac{\pi}{2}} \dot{\alpha}_{i}(t), \quad \forall t \in T .
$$

Note that we use the notation $\Delta J_{\mu}(\theta)$ to indicate that it is a function of $\theta$. We next compute the optimal twist $\theta^{*}$ such that $\Delta J_{\mu}(\theta)$ is minimized. $\theta$ is subject to the constraint that $\theta\left(t_{0}\right)=0, \theta\left(t_{f}\right)=2 k \pi$ for some fixed $k \in \mathbb{Z}$. For $\dot{\theta}$, this translates into $\int_{t_{0}}^{t_{f}} \dot{\theta}(t) d t=2 k \pi$. We can then write the Lagrangian function for this problem as

$$
\begin{aligned}
\mathcal{L}(\theta, \lambda) & \triangleq \Delta J_{\mu}(\theta)+\lambda\left[\int_{t_{0}}^{t_{f}} \dot{\theta}(t) d t-2 k \pi\right] \\
& =\int_{t_{0}}^{t_{f}}\left\{f(t)\left[\dot{\theta}(t)+\frac{g(t)+\frac{\lambda}{2}}{f(t)}\right]^{2}-\frac{\left[g(t)+\frac{\lambda}{2}\right]^{2}}{f(t)}\right\} d t-2 \lambda k \pi .
\end{aligned}
$$

Thus $\dot{\theta}^{*}(t)=-\left[g(t)+\lambda^{*} / 2\right] / f(t)$ where, since $\int_{t_{0}}^{t_{f}} \dot{\theta}(t) d t=2 k \pi, \lambda^{*}$ is given by:

$$
\lambda^{*}=-2\left[\int_{t_{0}}^{t_{f}} \frac{g(t)}{f(t)} d t+2 k \pi\right] / \int_{t_{0}}^{t_{f}} \frac{1}{f(t)} d t .
$$

Then, we have the following expression for $\dot{\theta}^{*}(t)$

$$
\dot{\theta}^{*}(t)=-\frac{g(t)}{f(t)}+\left[\int_{t_{0}}^{t_{f}} \frac{g(t)}{f(t)} d t+2 k \pi\right] /\left[f(t) \int_{t_{0}}^{t_{f}} \frac{1}{f(t)} d t\right] .
$$

Substituting this into equation (A.1), we get the minimal $\Delta J_{\mu}(\theta)$ :

$$
\Delta J_{\mu}\left(\theta^{*}\right)=\left[\int_{t_{0}}^{t_{f}} \frac{g(t)}{f(t)} d t+2 k \pi\right]^{2} / \int_{t_{0}}^{t_{f}} \frac{1}{f(t)} d t-\int_{t_{0}}^{t_{f}} \frac{g^{2}(t)}{f(t)} d t .
$$


If $\alpha=\alpha^{*}$ is an optimal maneuver, then $\Delta J_{\mu}\left(\theta^{*}\right) \geq 0$. Hence,

$$
\left[\int_{t_{0}}^{t_{f}} \frac{g(t)}{f(t)} d t+2 k \pi\right]^{2} \geq \int_{t_{0}}^{t_{f}} \frac{1}{f(t)} d t \cdot \int_{t_{0}}^{t_{f}} \frac{g^{2}(t)}{f(t)} d t
$$

In the case when $k=0$, the equality holds in equation (A.3) since the lower bound $\Delta J_{\mu}\left(\theta^{*}\right) \geq 0$ can be strictly achieved by choosing $\theta^{*}(t) \equiv 0$. Therefore,

$$
\left[\int_{t_{0}}^{t_{f}} \frac{g(t)}{f(t)} d t\right]^{2}=\int_{t_{0}}^{t_{f}} \frac{1}{f(t)} d t \cdot \int_{t_{0}}^{t_{f}} \frac{g^{2}(t)}{f(t)} d t
$$

Applying the Cauchy-Schwartz inequality to functions $1 / \sqrt{f(t)}$ and $g(t) / \sqrt{f(t)}$, we have that the above equality holds if and only if $g(t) / \sqrt{f(t)}=C / \sqrt{f(t)}$ for some constant $C$, i.e., if and only if $g(t) \equiv C$. In this case, equation (A.3) degenerates into:

$$
(C z+2 k \pi)^{2} \geq C^{2} z^{2}, \quad \forall k \in \mathbb{Z},
$$

where $z=\int_{t_{0}}^{t_{f}} 1 / f(t) d t$, or equivalently, $k \pi C z+k^{2} \pi^{2} \geq 0$ for all $k \in \mathbb{Z}$. This is possible if and only if $-\pi \leq C z \leq \pi$, thus completing the proof for the case $s=0$.

The general case when $s \neq 0$ can be reduced to the above case by first noticing that the optimality of $\alpha^{*}$ in $\mathbf{P}(R, \mathbf{a}, \mathbf{b})$ implies the optimality of $\alpha^{*}-s=\left(\alpha_{1}^{*}-s, \cdots, \alpha_{n}^{*}-s\right)$ in $\mathbf{P}(R, \mathbf{a}-s, \mathbf{b}-s)$, and then applying the results proved for the case $s=0$ to the optimal maneuver $\alpha^{*}-s$.

Appendix B. Geometry of $\mathbb{T}^{2}$ under metric $g$.

In Section 3.6, we define a Riemannian metric $g$ on the 2-torus $\mathbb{T}^{2}$. Here we will derive some useful quantities characterizing its geometry.

At each point $\left(\theta_{1}, \theta_{2}\right) \in \mathbb{T}^{2}$, a basis $\frac{\partial}{\partial \theta_{1}}$ and $\frac{\partial}{\partial \theta_{2}}$ of the tangent space of $\mathbb{T}^{2}$ is mapped by the differential of the coordinate map $f$ defined in (3.16) to

$$
\left\{\begin{array}{l}
d f\left(\frac{\partial}{\partial \theta_{1}}\right)=\frac{1}{3}\left(-2 \sin \theta_{1}, 2 \cos \theta_{1}, \sin \theta_{1},-\cos \theta_{1}, \sin \theta_{1},-\cos \theta_{1},\right)^{T}, \\
d f\left(\frac{\partial}{\partial \theta_{2}}\right)=\frac{1}{3}\left(\sin \theta_{2},-\cos \theta_{2},-2 \sin \theta_{2}, 2 \cos \theta_{2}, \sin \theta_{2},-\cos \theta_{2}\right)^{T},
\end{array}\right.
$$

which is a basis of the tangent space of $Q$ at $f\left(\theta_{1}, \theta_{2}\right)$. Here we have identified the tangent space of $\mathbb{R}^{6}$ at $f\left(\theta_{1}, \theta_{2}\right)$ with $\mathbb{R}^{6}$ itself, and the tangent space of $Q$ at $f\left(\theta_{1}, \theta_{2}\right)$ becomes a subspace of $\mathbb{R}^{6}$. The standard metric of $\mathbb{R}^{6}$ induces by $f$ isometrically the metric $g$ on $\mathbb{T}^{2}$ of the form:

$$
g=\left[\begin{array}{ll}
g_{11} & g_{12} \\
g_{21} & g_{22}
\end{array}\right]=\frac{1}{3}\left[\begin{array}{cc}
2 & -\cos \left(\theta_{1}-\theta_{2}\right) \\
-\cos \left(\theta_{1}-\theta_{2}\right) & 2
\end{array}\right],
$$

where $g_{i j} \triangleq\left\langle\frac{\partial}{\partial \theta_{i}}, \frac{\partial}{\partial \theta_{j}}\right\rangle$ for $i=1,2, j=1,2$. The inverse of $g$ can be written as

$$
g^{-1}=\left[\begin{array}{ll}
g^{11} & g^{12} \\
g^{21} & g^{22}
\end{array}\right]=\frac{3}{4-\cos ^{2}\left(\theta_{1}-\theta_{2}\right)}\left[\begin{array}{cc}
2 & \cos \left(\theta_{1}-\theta_{2}\right) \\
\cos \left(\theta_{1}-\theta_{2}\right) & 2
\end{array}\right]
$$

The covariant derivative $\nabla$ of $\mathbb{T}^{2}$ with respect to the Levi-Civita connection is defined by $([8])$

$$
\nabla_{\frac{\partial}{\partial \theta_{i}}} \frac{\partial}{\partial \theta_{j}}=\sum_{m=1}^{2} \Gamma_{i j}^{m} \frac{\partial}{\partial \theta_{m}}, \quad \forall 1 \leq i, j \leq 2,
$$


where $\Gamma_{i j}^{m}, 1 \leq i, j, m \leq 2$, are the Christoff symbols that can be computed by

$$
\Gamma_{i j}^{m}=\frac{1}{2} \sum_{k=1}^{2}\left\{\frac{\partial g_{j k}}{\partial \xi_{i}}+\frac{\partial g_{k i}}{\partial \xi_{j}}-\frac{\partial g_{i j}}{\partial \xi_{k}}\right\} g^{k m}, \quad 1 \leq i, j, m \leq 2 .
$$

It is easy to verify that

$$
\Gamma_{11}^{1}=-\Gamma_{22}^{2}=\frac{\sin \left(\theta_{1}-\theta_{2}\right) \cos \left(\theta_{1}-\theta_{2}\right)}{4-\cos ^{2}\left(\theta_{1}-\theta_{2}\right)}, \quad \Gamma_{11}^{2}=-\Gamma_{22}^{1}=\frac{2 \sin \left(\theta_{1}-\theta_{2}\right)}{4-\cos ^{2}\left(\theta_{1}-\theta_{2}\right)},
$$

and $\Gamma_{12}^{m}=\Gamma_{21}^{m}=0$ for $m=1,2$. The equations for geodesics in $\mathbb{T}^{2}$ are $\ddot{\xi}_{k}+$ $\sum_{i, j} \Gamma_{i j}^{k} \dot{\xi}_{i} \dot{\xi}_{j}=0, k=1,2$, which yield

$$
\begin{aligned}
& {\left[4-\cos ^{2}\left(\theta_{1}-\theta_{2}\right)\right] \ddot{\theta}_{1}=-\sin \left(\theta_{1}-\theta_{2}\right) \cos \left(\theta_{1}-\theta_{2}\right)\left(\dot{\theta}_{1}\right)^{2}+2 \sin \left(\theta_{1}-\theta_{2}\right)\left(\dot{\theta}_{2}\right)^{2}} \\
& {\left[4-\cos ^{2}\left(\theta_{1}-\theta_{2}\right)\right] \ddot{\theta}_{2}=-2 \sin \left(\theta_{1}-\theta_{2}\right)\left(\dot{\theta}_{1}\right)^{2}+\sin \left(\theta_{1}-\theta_{2}\right) \cos \left(\theta_{1}-\theta_{2}\right)\left(\dot{\theta}_{2}\right)^{2} .}
\end{aligned}
$$

The above equation are readily seen to be equivalent to equation (3.17).

Next, we will compute the curvature of $\mathbb{T}^{2}$. Let $R$ be the curvature tensor of $\mathbb{T}^{2}$. Let $R_{i j k l}$ be its value in basis $\frac{\partial}{\partial \theta_{1}}, \frac{\partial}{\partial \theta_{2}}$ defined by ([8])

$$
\begin{aligned}
R_{i j k l} & \triangleq\left\langle R\left(\frac{\partial}{\partial \theta_{i}}, \frac{\partial}{\partial \theta_{j}}\right) \frac{\partial}{\partial \theta_{k}}, \frac{\partial}{\partial \theta_{l}}\right\rangle \\
& =\left\langle\left(\nabla_{\frac{\partial}{\partial \theta_{j}}} \nabla_{\frac{\partial}{\partial \theta_{i}}}-\nabla_{\frac{\partial}{\partial \theta_{i}}} \nabla_{\frac{\partial}{\partial \theta_{j}}}+\nabla_{\left[\frac{\partial}{\partial \theta_{i}}, \frac{\partial}{\partial \theta_{j}}\right]}\right) \frac{\partial}{\partial \theta_{k}}, \frac{\partial}{\partial \theta_{l}}\right\rangle,
\end{aligned}
$$

for all $1 \leq i, j, k, l \leq 2$. Then $R_{i j k l}=\sum_{s=1}^{2} R_{i j k}^{s} g_{s l}$, where $R_{i j k}^{s}$ can be computed by

$$
R_{i j k}^{s}=\sum_{m=1}^{2} \Gamma_{i k}^{m} \Gamma_{j m}^{s}-\sum_{m=1}^{2} \Gamma_{j k}^{m} \Gamma_{i m}^{s}+\frac{\partial}{\partial \theta_{j}} \Gamma_{i k}^{s}-\frac{\partial}{\partial \theta_{i}} \Gamma_{j k}^{s} .
$$

In our case, calculation shows that

$$
R_{121}^{1}=R_{122}^{2}=\frac{-3 \cos ^{2}\left(\theta_{1}-\theta_{2}\right)}{\left[4-\cos ^{2}\left(\theta_{1}-\theta_{2}\right)\right]^{2}}, \quad R_{121}^{2}=R_{122}^{1}=\frac{-6 \cos \left(\theta_{1}-\theta_{2}\right)}{\left[4-\cos ^{2}\left(\theta_{1}-\theta_{2}\right)\right]^{2}},
$$

and $R_{21 k}^{s}=-R_{12 k}^{s}, R_{11 k}^{s}=R_{22 k}^{s}=0$ for all $1 \leq k, s \leq 2$. Hence,

$$
R_{1212}=\frac{-\cos \left(\theta_{1}-\theta_{2}\right)}{4-\cos ^{2}\left(\theta_{1}-\theta_{2}\right)}
$$

Therefore, the sectional curvature of $\mathbb{T}^{2}$ is

$$
K=\frac{R_{1212}}{g_{11} g_{22}-g_{12}^{2}}=\frac{-9 \cos \left(\theta_{1}-\theta_{2}\right)}{\left[4-\cos ^{2}\left(\theta_{1}-\theta_{2}\right)\right]^{2}} .
$$

$K$ depends only on $\theta_{1}-\theta_{2}$ since the map $\left(\theta_{1}, \theta_{2}\right) \mapsto\left(\theta_{1}+\xi, \theta_{2}+\xi\right) \bmod 2 \pi$ is an isometry of $\mathbb{T}^{2}$ for each $\xi$. In the special case when $\theta_{1}-\theta_{2}=\pi$, we have $K=1$. For further analysis, see [17]. 
[1] A. Abrams, Configuration Spaces and Braid Groups of Graphs, PhD thesis, University of California, Berkeley, 2000.

[2] F. Albrecht And I. Berg, Geodesics in Euclidean space with analytic obstacle, Proceedings of Amer. Math. Soc., 113 (1991), pp. 201-207.

[3] V. I. Arnold, K. Vogtmann, and A. Weinstein, Mathematical Methods of Classical Mechanics, Springer-Verlag, New York, 2nd ed., 1989.

[4] B. Aronov, M. de Berg, A. van der Stappen, P. Svestka, and J. Vleugels, Motion planning for multiple robots, Discrete \& Computational Geometry, 22 (1999), pp. 502-525.

[5] A. Bicchi and L. Pallottino, On optimal cooperative conflict resolution for air traffic management systems, IEEE Trans. on Intelligent Transport. Systems, 1 (2000), pp. 221-231.

[6] J. Birman, Braids, Links, and Mapping Class Groups, Princeton University Press, Princeton, NJ, 1974.

[7] J. P. Desai And V. Kumar, Nonholonomic motion planning for multiple mobile manipulators, in Proc. IEEE Int. Conf. on Robotics and Automation, vol. 4, IEEE, 1997, pp. 3409-3414.

[8] M. P. do Carmo, Riemannian Geometry, Birkhäuser, Boston, MA, 1992.

[9] M. Erdmann and T. Lozano-Perez, On multiple moving objects (motion planning), Algorithmica, 2 (1987), pp. 477-521.

[10] E. Frazzoli, Z.-H. MaO, J.-H. Оh, and E. Feron, Resolution of conflicts involving many aircraft via semidefinite programming, J. of Guidance, Control, and Dynamics, 24 (2001), pp. $79-86$.

[11] K. FujImura, Motion Planning in Dynamic Environments, Springer-Verlag, Tokyo, 1991.

[12] J. Hu, A study of conflict detection and resolution in free flight, master's thesis, University of California, Berkeley, 1999.

[13] J. Hu, M. Prandini, K. H. Johansson, and S. Sastry, Hybrid geodesics as optimal solutions to the collision-free motion planning problem, in Hybrid Systems: Computation and Control. 4th International Workshop (HSCC01), M. Domenica, D. Benedetto, and A. Sangiovanni-Vincentelli, eds., vol. 2034 of Lecture Notes in Computer Science, Rome, Italy, March 2001, Springer-Verlag, Berlin, pp. 305-318.

[14] J. Hu, M. Prandini, AND S. SAStRY, Optimal maneuver for multiple aircraft conflict resolution: a braid point of view, in Proc. 39th IEEE Int. Conf. on Decision and Control, vol. 4, IEEE, 2000, pp. 4164-4169.

[15] — Optimal coordinated maneuvers for three dimensional aircraft conflict resolution, J. of Guidance, Control, and Dynamics, (2002). To appear.

[16] J. Hu AND S. SASTRY, Optimal collision avoidance and formation switching on Riemannian manifolds, in Proc. 40th IEEE Int. Conf. on Decision and Control, vol. 2, IEEE, 2001, pp. 1071-1076.

[17] - Hybrid geodesic flows on manifolds with boundary, tech. report, University of California, Berkeley, in preparation, 2002.

[18] Y. K. Hwang And N. AhujA, Gross motion planning, ACM Computing Surveys, 24 (1992), pp. 219-291.

[19] A. Inselberg and B. Dimsdale, Multidimensional lines II: proximity and applications, SIAM J. on Applied Mathematics, 54 (1994), pp. 578-596.

[20] J. Jost, Riemannian Geometry and Geometric Analysis, Springer-Verlag, Berlin, 2nd ed., 1998.

[21] W.-S. Koon And J. E. MARsden, Optimal control for holonomic and nonholonomic mechanical systems with symmetry and Lagrangian reduction, SIAM J. on Control and Optimization, 35 (1997), pp. 901-929.

[22] P. S. Krishnaprasad, Eulerian many-body problems, in Dynamics and Control of Multibody Systems (Brunswick, ME, 1988), Providence, RI, 1989, Amer. Math. Soc., pp. 187-208.

[23] J. Kuchar And L. C. YAng, Survey of conflict detection and resolution modeling methods, IEEE Trans. on Intelligent Transport. Systems, 1 (2000), pp. 179-189.

[24] J. Latombe, Robot Motion Planning, Kluwer Academic, Boston, MA, 1991.

[25] S. LaValle and S. Hutchinson, Optimal motion planning for multiple robots having independent goals, IEEE Trans. on Robotics and Automation, 14 (1998), pp. 912-925.

[26] J. E. Mardden And T. S. Ratiu, Introduction to Mechanics and Symmetry, Springer-Verlag, New York, 2nd ed., 1999.

[27] C. D. Medio And G. Oriolo, Robot obstacle avoidance using vortex fields, in Advances in Robot Kinematics, S. Stifter and J. Lenarcic, eds., Springer-Verlag, Wien, 1991, pp. 227235.

[28] F. Medioni, N. Durand, and J. M. Alliot, Air traffic conflict resolution by genetic algorithms, in Artificial Evolution, European Conference (AE 95), Springer-Verlag, Berlin, 1995, pp. 370-383. 
[29] P. Menon, G. Sweriduk, And B. Sridhar, Optimal strategies for free-flight air traffic conflict resolution, J. of Guidance, Control, and Dynamics, 22 (1999), pp. 202-211.

[30] A. Miele, T. Wang, C. Chao, and J. Dabney, Optimal control of a ship for collision avoidance maneuvers, J. of Optimization Theory and App., 103 (1999), pp. 495-519.

[31] J. W. Milnor, Morse Theory, Princeton University Press, Princeton, NJ, 1963.

[32] S. Morgan, The Mathematical Theory of Knots and Braids: an Introduction, North-Holland, Amsterdam, 1991.

[33] K. Murasugi and B. I. Kurpita, A Study of Braids, Kluwer Academic, Boston, MA, 1999.

[34] M. Prandini, J. Hu, J. Lygeros, and S. Sastry, A probabilistic approach to aircraft conflict detection, IEEE Trans. on Intelligent Transport. Systems, 1 (2000), pp. 199-220.

[35] Radio Technical Commission for Aeronautics, Minimum aviation system performance standards for automatic dependent surveillance-broadcast (ADS-B), Technical report, RTCA-186 (1997). DRAFT 4.0.

[36] E. Rimon AND D. KoditscheK, Exact robot navigation using artificial potential functions, IEEE Trans. on Robotics and Automation, 8 (1993), pp. 501-519.

[37] M. RudE, Collision avoidance by using space-time representations of motion processes, Autonomous Robots, 4 (1997), pp. 101-119.

[38] M. Sharir And S. Sifrony, Coordinated motion planning for two independent robots, Ann. Math. Artificial Intelligence, 3 (1991), pp. 107-130.

[39] C. Tomlin, Hybrid control of air traffic management systems, PhD thesis, University of California, Berkeley, 1998.

[40] C. Tomlin, G. Pappas, And S. Sastry, Conflict resolution for air traffic management: a study in multi-agent hybrid systems, IEEE Trans. on Automatic Control, 43 (1998), pp. 509-521. 